\title{
1 Novel genetic basis of resistance to Bt toxin Cry1Ac in Helicoverpa zea
}

3 Kyle M. Benowitz ${ }^{1,2,}{ }^{,}$, Carson W. Allan ${ }^{1}$, Benjamin A. Degain ${ }^{1}$, Xianchun Li ${ }^{1}$, Jeffrey A. Fabrick ${ }^{3}$,

4 Bruce E. Tabashnik ${ }^{1}$, Yves Carrière ${ }^{1}$, Luciano M. Matzkin ${ }^{1,4,5}$

$5 \quad{ }^{1}$ Department of Entomology, University of Arizona, Tucson, AZ, USA

$6 \quad{ }^{2}$ Department of Biology, Austin Peay State University, Clarksville, TN, USA

$7 \quad{ }^{3}$ U.S. Department of Agriculture, Agricultural Research Service, U.S. Arid Land Agricultural Research Center,

8 Maricopa, AZ, USA

$9 \quad{ }^{4}$ Department of Ecology and Evolutionary Biology, University of Arizona, Tucson, AZ, USA

$10{ }^{5}$ Bio5 Institute, University of Arizona, Tucson, AZ, USA

11

12 Corresponding author: Kyle M. Benowitz. Department of Biology, Austin Peay State University, Sundquist Science

13 Center, PO Box 4718, Clarksville, TN 37044. Email: benowitzk@apsu.edu

14

15

16 Running Title: Novel genetic basis of Bt resistance 


\section{$17 \quad$ Abstract}

18 Crops genetically engineered to produce insecticidal proteins from the bacterium Bacillus

19 thuringiensis $(\mathrm{Bt})$ have advanced pest management, but their benefits are diminished when

20 pests evolve resistance. Elucidating the genetic basis of pest resistance to Bt toxins can improve

21 resistance monitoring, resistance management, and design of new insecticides. Here, we

22 investigated the genetic basis of resistance to Bt toxin Cry1Ac in the lepidopteran Helicoverpa

$23 z e a$, one of the most damaging crop pests in the United States. To facilitate this research, we

24 built the first chromosome-level genome assembly for this species, which has 31 chromosomes

25 containing $375 \mathrm{Mb}$ and 15,482 predicted proteins. Using a genome-wide association study, fine-

26 scale mapping, and RNA-seq, we identified a 250-kb quantitative trait locus (QTL) on

27 chromosome 13 that was strongly associated with resistance in a strain of $H$. zea that had been

28 selected for resistance in the field and lab. The mutation in this QTL contributed to but was not

29 sufficient for resistance, which implies alleles in more than one gene contributed to resistance.

30 This QTL contains no genes with a previously reported role in resistance or susceptibility to Bt

31 toxins. However, in resistant insects, this QTL has a premature stop codon in a kinesin gene

32 which is a primary candidate as a mutation contributing to resistance. We found no changes in

33 gene sequence or expression consistently associated with resistance for 11 genes previously

34 implicated in lepidopteran resistance to Cry1Ac. Thus, the results reveal a novel and polygenic

35 basis of resistance.

37 Keywords: Bacillus thuringiensis; genome wide association study; genome assembly; insecticide

38 resistance; kinesin; Lepidoptera; transgenic crops 


\section{Introduction}

42 Crops genetically engineered to produce insecticidal proteins from Bacillus thuringiensis (Bt)

43 have provided control of some key pests during the past 25 years while reducing insecticide

44 sprays and conserving arthropod natural enemies (Bravo et al. 2011; NASEM 2016; Dively et al.

45 2018; Romeis et al. 2018; Carrière et al. 2020a; Tabashnik et al. 2021). However, planting of a

46 cumulative total of more than one billion hectares of Bt crops worldwide (ISAAA 2019) has

47 selected for resistance that has reduced the efficacy of Bt crops against populations of at least

48 nine major pest species (Calles-Torrez et al. 2019; Smith et al. 2019; Tabashnik and Carrière

49 2019). Knowledge of the genetic basis of pest resistance to Bt toxins can be useful for improving

50 monitoring and management of resistance, as well as for designing new insecticides (Soberón et

51 al. 2007; Jin et al. 2018).

52 Resistance to crystalline (Cry) Bt toxins typically entails mutations that reduce binding of the

53 toxins to larval midgut receptors and thus block an essential step in the mode of action (Heckel et

54 al. 2007; Peterson et al. 2017; Jurat-Fuentes et al. 2021). In particular, research has repeatedly

55 implicated disruption or reduced expression of known or putative Bt toxin receptors from four

56 protein families: ATP-binding cassette (ABC), cadherin, aminopeptidase N (APN), and alkaline

57 phosphatase (ALP). Mutations that disrupt binding of toxins to receptors are frequently

58 associated with high levels of resistance to one or a few closely related Bt toxins, weak or no

59 cross-resistance to unrelated Bt toxins, and recessive inheritance of resistance (Mode 1

60 resistance; Tabashnik et al. 1998). Nonetheless, lepidopteran resistance to Bt toxins also includes

61 examples where proteins from these families are not involved, toxin binding is not reduced, and

62 inheritance of resistance is not recessive (Peterson et al. 2017; Jin et al. 2018).

63 Here we analyzed the genetic basis of resistance to Bt toxin Cry1 Ac in the lepidopteran

64 Helicoverpa zea (corn earworm or bollworm), which is one of the most economically important

65 crop pests in the United States (Cook and Threet 2019; Musser et al. 2019). This polyphagous

66 pest is the first insect reported to have evolved resistance to a Bt crop, specifically to cotton

67 producing Cry1Ac (Luttrell et al. 1999; Ali et al. 2006; Tabashnik et al. 2008; Reisig et al.

68 2018). In contrast with Mode 1 resistance, inheritance of resistance to Cry1Ac in H. zea is not

69 completely recessive (Brévault et al. 2013, 2015; Carrière et al. 2020b; Reisig et al. 2021). 
Caccia et al. (2012) concluded that resistance in their lab-selected AR1 strain of H. zea was complex, possibly polygenic, and not caused primarily by reduced binding of Cry1 Ac to larval

72 midgut membranes. Perera et al. (2021) found that knocking out the gene encoding the putative

73 Cry1 Ac receptor ABCC2 increased the concentration of Cry1 Ac killing 50\% of larvae (LC50) by

74 7- to 40-fold. Because >100-fold resistance to Cry1Ac is common in lab- and field-selected $H$.

75 zea (Caccia et al. 2012; Brévault et al. 2013; Reisig et al. 2018; Kaur et al. 2019), they inferred

76 that mutations disrupting $\mathrm{ABCC} 2$ are not the sole or primary mechanism of resistance to Cry1 Ac

77 in H. zea. Although most previous studies of Bt resistance in H. zea have emphasized the gene

78 families listed above (Caccia et al. 2012; Zhang et al. 2019a; Fritz et al. 2020; Perera et al. 2021;

79 Taylor et al. 2021), additional candidates have been identified using RNA-seq (Lawrie et al.

$802020 ; 2022)$.

81 Our work focuses on the GA-R strain of $H$. zea, which had been selected for resistance to $\mathrm{Bt}$ 82 toxins in the field and lab (Brévault et al. 2013, Welch et al. 2015). GA-R was derived from the

83 moderately resistant GA strain, which had been selected for resistance to $\mathrm{Bt}$ toxins only in the

84 field (Brévault et al. 2013). Relative to an unrelated susceptible lab strain (LAB-S) of H. zea, the

85 LC50 of Cry1 Ac was $>500$ times higher for GA-R and 55 times higher for GA (Brévault et al.

86 2013). Previous work identified reduced activation of Cry 1 Ac by midgut proteases as a potential

87 field-selected mechanism of resistance that could explain part but not all of the resistance in GA-

88 R relative to LAB-S (Zhang et al. 2019a). Overall, the previous results with GA-R and other

89 strains of $H$. zea summarized above led us to hypothesize that resistance to Cry1 Ac in this

90 species is polygenic and entails novel genetic mechanisms. Accordingly, genome-wide mapping

91 approaches are warranted, but have been hindered because the only $H$. zea genome assembly

92 available is highly fragmented (Pearce et al. 2017).

93 Here, we generated a chromosome-level genome for $\mathrm{H}$. zea, then used a genome wide

94 association study (GWAS), fine-scale mapping, and RNA-seq to elucidate the genetic basis of

95 resistance to Cry1Ac in the GA-R strain of H. zea. We identified a quantitative trait locus (QTL)

96 of $250 \mathrm{~kb}$ on chromosome 13 that was strongly associated with resistance to Cry1 Ac. The results

97 indicate a mutation in this QTL contributed to resistance but was not sufficient for resistance in

98 GA-R. We also found no consistent association between resistance to Cry1Ac and any of 11

99 genes previously implicated in lepidopteran resistance to Bt toxins. We conclude the genetic 
100 basis of resistance to Cry1Ac in GA-R is novel and polygenic.

101

102

103

104

105

106

107

108

109

110

\section{Materials and methods}

\section{Insect strains} with Cry1 Ab, or both.

We used four strains of $H$. zea: the highly resistant strain GA-R, its moderately resistant parent strain GA, the unrelated susceptible strain LAB-S, and the heterogeneous strain GA-RS that we created by crossing GA-R with LAB-S as described below. LAB-S was obtained from Benzon Research Inc. (Carlisle, PA, USA) and has not been exposed to Bt toxins or other insecticides. The resistant strain GA-R was derived from the third generation (F3) of the GA strain, which was started with 180 larvae collected on Cry1Ab corn from Tifton, Georgia in 2008 (Brévault et al., 2013). GA-R was initially selected with Cry1Ac for nine generations and with MVPII thereafter (Brévault et al. 2013; Fritz et al. 2020; Carrière et al. 2020b). MVPII is a liquid formulation of a hybrid protoxin produced by transgenic Pseudomonas fluorescens. The amino acid sequence of the active portion of the protoxin is identical in the hybrid protoxin and Cry1Ac (Welch et al. 2015). For simplicity, hereafter we refer to MVPII as Cry1Ac. Amino acid sequence similarity between Cry1Ab and Cry1 Ac is $86 \%$ (Carrière et al. 2015). Lab selection with Cry1Ac caused cross-resistance to Cry1Ab in GA-R (Welch et al. 2015) and in the AR strain of H. zea (Anilkumar et al. 2008). Moreover, adoption of Cry1Ac-producing cotton, a host plant of $H$. zea, was 94\% in Georgia in 2008 (USDA 2008) and high in several preceding years (USDA 2020). Thus, the observed resistance to Cry1Ac in the GA strain (Brévault et al. 2013) could reflect direct selection in the field with Cry1Ac, cross-resistance from selection in the field

We conducted all rearing in walk-in growth chambers at $27 \pm 1^{\circ} \mathrm{C}$ with $14 \mathrm{~h}$ light: $10 \mathrm{~h}$ dark photoperiod. We reared larvae on a casein-based wheat germ diet (Orpet et al. 2015a, 2015b) and conducted larval bioassays on Southland diet (Southland Products, Inc., Lake Village, AR, USA). We use these two different diets to optimize larval development and surface uniformity for toxin overlay in bioassays, respectively (Carrière et al. 2020b). Moths were kept in walk-in growth chambers under the same temperature and photoperiod mentioned above but under higher 
128

129

130

131

132

133

134

135

136

137

138

139

140

141

142

143

144

145

146

147

relative humidity than for larvae (60\% $\mathrm{Rh}$ for moths and $20 \% \mathrm{Rh}$ for larvae). Moths had access to a $10 \%$ sugar water solution for feeding and cheese cloth for oviposition (Welch et al. 2015). As previously reported (Fritz et al. 2020), we reared ca. 600 moths per generation for the first 35 and 33 generations of GA and GA-R, respectively. In 2012, we crossed GA with GA-R and used the resulting progeny to continue GA-R (Carrière et al. 2020b). After this interstrain cross, to reduce genetic drift and inbreeding, we reared two subsets of GA and crossed the two subsets every one to three generations (Carrière et al. 2020b). We used the same procedure to rear and cross two subsets of GA-R. Each subset had ca. 600 moths per generation (ca. 1200 moths per strain per generation). For GA, the mean number of moths per generation for F1 to F72 was ca. 900, based on the number of moths per generation of 600 for F1-F36 and 1200 for F37-72.

Relative to GA, GA-R had significantly higher survival on Bt cotton (producing Cry1Ac, Cry1Ac + Cry2Ab, or Cry1Ac + Cry1F) and Bt corn producing Cry1A.105 + Cry2Ab (Brévault et al. 2013, 2015; Carrière et al. 2018, 2019, 2020b, 2021). At the time we crossed GA-R with LAB-S in May 2018, we had selected GA-R with Cry1Ac for 58 generations. The GA-RS strain was created using mass reciprocal crosses between GA-R and LAB-S (i.e., 120 GA-R females $\times$ 120 LAB-S males and 120 LAB-S females $\times 120$ GA-R males). GA-RS was founded with 450 neonates from each reciprocal cross. In the founding and subsequent generations, 900 larvae were reared and 600 adults (sex ratio 1:1) produced the neonates used for propagating the next generation.

\section{Genome sequencing of resistant strain GA-R}

We generated a de novo genome assembly for GA-R using an approach combining a hybrid short- and long-read assembly with a long-read only assembly (Jaworski et al. 2020), which allows for improved error correction of long read data (Ye et al. 2016) without the need for massive coverage (Chakraborty et al. 2016). Hybrid assembly strategies have been used frequently with error-prone PacBio CLR data to generate highly contiguous genomes in nonmodel insect species (Hartke et al. 2019; Wan et al. 2019; Ferguson et al. 2020; Jaworski et al.

2020; Ma et al. 2020; Mathers 2020; Schmidt et al. 2020; Xu et al. 2021). For the short-read 
156

157

158

159

160

161

162

163

164

165

166

167

168

169

170

171

172

173

174

175

176

177

178

179

180

181

182

183

184

185

assembly, we collected and sequenced 30 GA-R larvae as described below. We trimmed reads and generated the assembly using Platanus (Kajitani et al. 2014). For the long-read assembly, we extracted DNA from the gut of a single GA-R fifth instar using a chloroform-based extraction method (Jaworski et al. 2020). PacBio libraries were constructed at the Arizona Genomics Institute (Tucson, AZ, USA). We then sequenced the library on a single lane of PacBio Sequel II, also at the Arizona Genomics Institute. We formatted raw PacBio reads using bam2fastq (https://github.com/jts/bam2fastq) and SeqKit (Shen et al. 2016) before filtering out all reads under 30-kb using Filtlong (https://github.com/rrwick/Filtlong). We mapped contigs from the short-read assembly to the long reads using DBG2OLC (Ye et al. 2016) before running three iterations of Sparc (Ye and Ma 2016) to correct the resulting contigs. We realigned the raw PacBio reads to the resulting assembly with pbmm2

(https://github.com/PacificBiosciences/pbmm2) and polished contigs using Arrow (Chin et al. 2013; https:/github.com/PacificBiosciences/GenomicConsensus). Lastly, we mapped raw short reads to the assembly with Bowtie2 (Langmead and Salzberg 2012) to perform a final polishing step using Pilon (Walker et al. 2014).

We generated the PacBio-only assembly with Canu (Koren et al. 2017), using the reads longer than $30 \mathrm{~kb}$ after filtering described above. We then polished the assembly using Arrow and Pilon as described above. Finally, we used Purge Haplotigs (Roach et al. 2018) to remove contigs containing alternate haplotypes generated due to high heterozygosity.

We aligned the two assemblies using nucmer within MUMmer4 (Marçais et al. 2018), keeping only alignments greater than $10 \mathrm{~kb}$. We then generated the merged assembly using Quickmerge (Chakraborty et al. 2016). We performed additional merging by re-running Quickmerge with more liberal parameters on individual contig pairs after hypothesizing their contiguity based on synteny with H. armigera (Pearce et al. 2017; Valencia-Montoya et al. 2020). Specifically, chromosomes 5, 7, 8, 16, 17, 18, 19, 21, 23, 29, and 30 required such additional merging. After this step, only chromosome 17 had two contigs that did not merge. We therefore connected them with a default 100-bp gap according to NCBI standards (Karsch-Mizrachi et al. 2012). We again polished the final assembly using Arrow, Pilon, and Purge Haplotigs as above. Lastly, we ordered and named each chromosome to align with those of H. armigera. We generated a synteny plot comparing our genome to the $H$. armigera genome using Dot 
(https://github.com/marianattestad/dot) after filtering out alignments under 4000 bp in NUCmer.

187 We analyzed the completeness of the GA-R genome using BUSCO v.5 (Seppey et al. 2019),

188 comparing genomic content against the lepidodptera_odb10 set of 5,286 conserved single copy

189 genes. We calculated contig (before final merging of chromosome 17) and scaffold (final

190 assembly) genome summary statistics with bbmap stats

191 (https://sourceforge.net/projects/bbmap/). We calculated repeat content with RepeatModeler2

192 (Flynn et al. 2020) and RepeatMasker (Smit et al. 2013-2015). We provide a preliminary

193 annotation produced following the funannotate pipeline (Palmer and Stajich 2020) with

194 transcripts from $H$. armigera and proteins from $B$. mori used as evidence supporting putative

195 annotations. We also used BUSCO v.5 to assess the completeness and accuracy of the annotation 196 against the 5,286 single copy genes in the lepidoptera_odb10 dataset. To compare the quality of

197 our assembly and annotation with the previous H. zea genome assembly (Pearce et al. 2017), we

198 reanalyzed the genomic and proteomic BUSCO scores of that assembly against the same

199 lepidoptera_odb10 dataset.

\section{Phenotyping of Cry1Ac-susceptible and -resistant larvae for genetic mapping}

202 We used our standard diet overlay bioassay (Welch et al. 2015) to characterize GA-RS larvae as 203 susceptible or resistant to Cry1Ac. We added $40 \mu \mathrm{l}$ of a dilution containing $0.1 \%$ Triton X-100

204 and the desired concentration of Cry1Ac to the surface of solidified Southland diet in each well 205 of 128-well bioassay trays (C-D International, Pitman, NJ, USA). One neonate ( $<8$ h old) was 206 transferred to each well and trays were covered with ventilated plastic lids (C-D International) 207 and held for 7 days under the abiotic conditions mentioned above.

208 We conducted five sets of bioassays using GA-RS neonates from the F2 (July 2018), F12 (July 209 2019), F22 (June 2020), F23 (July 2020), and F26 (October 2020) generations (Supplementary

210 Table S1). Neonates were exposed to diet with 0 (control), 1, or $10 \mu \mathrm{g}$ Cryl Ac per $\mathrm{cm}^{2}$ diet.

211 After 7 days, live first instar larvae on diet with $1 \mu \mathrm{g}$ CrylAc per $\mathrm{cm}^{2}$ were considered

212 susceptible because this low toxin concentration inhibited their growth, whereas live larvae on 213 diet with $10 \mu \mathrm{g}$ Cry1Ac per $\mathrm{cm}^{2}$ that were third or subsequent instars were considered resistant 
214 because they grew well despite this high toxin concentration. For control larvae reared on diet

215 without Cry1Ac, mean survival to third instar was 96\% (range: 91-100\%, mean n = 99 larvae per

216 bioassay in five bioassays). Susceptible, resistant, and control larvae were transferred

217 individually to plastic cups containing non-Bt diet, reared to fifth instar, transferred individually

218 to $1.5 \mathrm{ml}$ plastic tubes, and frozen at $-80^{\circ} \mathrm{C}$ for subsequent genomic comparison.

220 Genomic comparison of GA-R and LAB-S

221 We sequenced pools of larvae from the parental strains GA-R and LAB-S, which allowed us to

222 evaluate genetic variation between and within these parental strains. This also allowed us to

223 check if SNPs associated with resistance in the GWAS of GA-RS were more common in GA-R,

224 and if those associated with susceptibility were more common in LAB-S. In April 2019, we

225 collected 30 third instars from each strain, extracted DNA using Qiagen DNeasy Blood and

226 Tissue Kits (Qiagen, Hilden, Germany), and constructed Illumina libraries using KAPA LTP

227 Library Preparation Kits (Roche, Basel, Switzerland). We sequenced both libraries on an

228 Illumina HiSeq4000 at Novogene (Beijing, China). We called variants with Platypus after read

229 trimming and alignment to the genome using Trimmomatic and bwa-mem as described above.

230 To detect potential selective sweeps in each strain, we used SAMtools mpileup (Li 2011) and

231 PoPoolation (Kofler et al. 2011) to calculate Tajima's D in 50-kb windows overlapping by $10 \mathrm{~kb}$

232 across the genome.

234 Genome-wide association study for Cry1Ac resistance

235 From the heterogeneous strain GA-RS F12 larvae phenotyped in July 2019, we extracted DNA

236 from 144 resistant and 144 susceptible larvae using ZYMO Quick-DNA 96 Plus Kits and

237 quantified the DNA concentration of each individual using a Nanodrop (Thermo Fisher

238 Scientific, Waltham, MA, USA). We combined equal amounts of DNA from each of the 144

239 resistant larvae to make a resistant pool and from each of the 144 susceptible larvae to make a

240 susceptible pool, then generated libraries using KAPA LTP Library Preparation Kits for each 
pool. We sequenced both libraries on an Illumina HiSeq4000 at Novogene (Beijing, China).

242 We demultiplexed reads and trimmed for adapter contamination and low-quality sequence using

243 Trimmomatic (Bolger et al. 2014). We mapped reads to the de novo H. zea genome using bwa-

244 mem (Li and Durbin 2009). We used Platypus (Rimmer et al. 2014) to call and quantify SNP

245 variants and short INDELs. We extracted biallelic SNPs with a minimum coverage of 20 in each

246 pool and a total coverage between 60 and 500 from the Platypus output for statistical analysis,

247 for which we used two approaches (Benowitz et al. 2019). First, we calculated a Z-statistic

248 (Huang et al. 2012), using the formula $Z=\frac{\rho_{1}-\rho_{2}}{\sqrt{\left(\rho_{0}\left(1-\rho_{0}\right)\left(\frac{1}{n}+\frac{1}{c_{1}}+\frac{1}{c_{2}}\right)\right.}}$, where $\rho_{1}$ and $\rho_{2}$ are the reference

249 alleles frequencies of each bulk, $\rho_{0}$ is the mean allele frequency across bulks, $n$ is the sample size

250 of each bulk, and $c_{1}$ and $c_{2}$ are the read depth of each bulk. We evaluated statistical significance

251 of $Z$ against the standard normal distribution. Following convention (Barsh et al. 2012, Welter et

252 al. 2014), we used $P<5 \times 10^{-8}$ as a threshold for significance. To estimate the density of

253 significant sites, we used the R package WindowScanR

254 (https://github.com/tavareshugo/WindowScanR) to calculate the percentage of SNPs with a more

255 liberal threshold of $P<10^{-5}$ in $10-\mathrm{kb}$ windows overlapping by $5 \mathrm{~kb}$. Density of significant sites

256 may be a particularly useful parameter because the magnitude of each individual $P$-value from a

257 bulk segregant analysis is highly sensitive to noise. Close linkage to the causal allele, however,

258 should result in a higher density of significant sites even if the $P$-value itself varies. Second, we

259 performed a sliding-window analysis with 500-kb windows overlapping by $250 \mathrm{~kb}$ using the $\mathrm{R}$

260 package QTLseqr (Mansfeld and Grumet 2018), which implements the G' method of Magwene

261 et al. (2011). This method provides a statistical determination of whether an entire QTL, rather

262 than an individual SNP, is significant, and also defines borders to QTLs deemed significant.

264 Fine-scale mapping within chromosome 13

265 Using larvae from the F22 and F23 generation (July 2020), we conducted fine-scale mapping 266 within the QTL in chromosome 13 associated with resistance, which we refer to hereafter as the $267 r 1$ locus. Using the methods described above, larvae were scored as resistant or susceptible. 
After phenotyping, we reared susceptible larvae to fifth instars on untreated diet before storing all larvae (62 resistant and 51 susceptible) at $-80^{\circ} \mathrm{C}$. We extracted DNA from each sample using

270 Qiagen DNeasy Blood and Tissue Kits and genotyped each larva individually via high-resolution

271 melt curve (HRM) analysis at 12 SNP marker sites within the chromosome 13 QTL

272 (Supplementary Table S2). For each site, we performed PCR in $10 \mu 1$ reactions using Apex Taq

273 DNA polymerase and EvaGreen Dye (Biotium, Fremont, CA, USA) as the intercalating dye. We

274 ran RT-PCR in a QuantStudio 3 Real-Time PCR machine (Thermo Fisher) using continuous

275 capture with a $0.025^{\circ} \mathrm{C} / \mathrm{s}$ ramp. We used QuantStudio Design and Analysis Software (Thermo

276 Fisher) to manually score melt curves for the identity of the focal SNP. We compared test melt

277 curves against control curves generated from the parental LAB-S and GA-R strains. We used

278 Fisher's exact test to assess significant differences between allele frequencies of resistant and

279 susceptible individuals.

280 The lack of amplification from some individuals for some markers yielded variation in sample 281 size among the 12 markers. These ranged from 40 to 60 for resistant larvae $($ mean $=56)$ and 20

282 to $50($ mean $=45)$ for susceptible larvae. We also used HRM to obtain genotypes at marker \#4

283 for 23 of 25 resistant larvae and 89 of 95 control larvae tested from the F26 generation. The

284 control larvae were not screened with a bioassay and thus contained a mixture of resistant and 285 susceptible individuals.

286 To confirm the accuracy of HRM genotyping, we Sanger sequenced a single site (marker 4;

287 Supplementary Table S2) for all 60 resistant and a subset of 34 susceptible individuals. We 288 designed new primers to produce a longer amplicon, and confirmed the quality of the resultant 289 amplicons with gel electrophoresis. We cleaned the PCR product with Exonuclease I and 290 Antarctic Phosphatase (New England BioLabs, Ipswich, MA, USA) before sending to Eurofins 291 Genomics (Louisville, KY, USA) for Sanger sequencing.

292 In addition to the fine mapping data from the HRM, we used the data on significant SNP density 293 from the original GWAS experiment as well as the Tajima's D results from comparison of the 294 GA-R and LAB-S strains to provide additional support for narrowing the region associated with 295 resistance within chromosome 13. 
We performed several analyses of genotype frequencies at marker 4 to understand how $r 1$ affects resistance. We used the genotypes from the RNA-seq study (see below) as a control group to examine the frequency of marker 4 in the GA-RS strain at the F26 generation. We also used a $\chi^{2}$ test to examine departure from Hardy-Weinberg equilibrium.

We used Fisher's exact test to determine if resistant individuals from GA-RS were more likely to be homozygous for alleles from GA-R (GG) than heterozygous with one allele from GA-R and the other from LAB-S (GL) by comparing the frequencies of each genotype in the resistant samples from generations F22, F23, and F26 to the control samples from generation F26. We calculated the dominance parameter $h$, which varies from 0 for recessively inherited resistance to 1 for dominantly inherited resistance (Liu and Tabashnik 1997), using the genotype frequencies at marker 4 in the F22, F23, and F26 for resistant, control, and susceptible larvae (Supplementary Table S3). The results from F22 and F23 were similar and were pooled to increase the sample size for analyses.

311 To evaluate the relationship between genotype and resistance, we compared their trajectories

312 across generations in GA-RS. We used linear regressions in R 4.1.0 to test the null hypothesis of

313 no change in the $\log _{10}$ of percentage survival to third instar. Bioassays used 1 or $10 \mu \mathrm{g}$ of Cry1Ac 314 per $\mathrm{cm}^{2}$ in generations F2, F22 and F23, and only the higher concentration in the F26 test.

\section{RNA-seq and candidate gene analysis}

317 To generate samples for RNA sequencing, we reared LAB-S, GA-R, and GA-RS (generation 26)

318 individuals on untreated diet as described above in October 2020. When the larvae of the

319 parental strains LAB-S and GA-R reached the third instar, we dissected midguts from 15

320 individuals and froze them in groups of five, generating three biological replicates per strain. For

321 the GA-RS heterogeneous strain, we dissected 95 third instar midguts and froze them 
322

323

324

325

326

327

328

329

330

individually, while simultaneously freezing the remainder of the carcass separately in wells of a PCR plate. We extracted DNA from each sample using a squish extraction (Gloor et al. 1993) using $50 \mu \mathrm{l}$ of buffer. We screened samples HRM as above at marker 4, which was one of two sites we found to be most strongly associated with resistance. We selected 15 individuals that were homozygous for the LAB-S genotype at this site (henceforth "LL") and 15 individuals homozygous for the GA-R genotype at this site (henceforth "GG") and pooled the midguts corresponding to these samples into groups of five, again generating three biological replicates for each genotype. Selecting genotypes in this way allowed us to isolate the effects that the chromosome 13 QTL has on gene expression, giving us the potential to detect trans-regulatory effects. We extracted RNA from all 12 midgut samples using ZYMO Direct-zol RNA Miniprep Kit kits and built paired-end libraries with KAPA stranded mRNA seq kits. Libraries were sequenced in part on an Illumina NextSeq at the University of Arizona Genetics Core (UAGC; Tucson, AZ, USA) and part on an Illumina NovaSeq at Novogene.

We trimmed reads using Trimmomatic (Bolger et al. 2014) and aligned them to our H. zea assembly with Hisat2 (Kim et al. 2019). We built genome-guided transcriptome assemblies for each sample using StringTie (Pertea et al. 2016) and used StringTie merge to create a single transcriptome. We used blastp to identify the closest ortholog in H. armigera for each gene. We quantified read abundance for each sample using Salmon (Patro et al. 2017) and combined its transcript-level counts into gene-level counts with the R package tximport (Soneson et al. 2016). We analyzed differential expression using FDR-corrected $P$-values from negative binomial models at the gene level with edgeR (Robinson et al. 2010), after filtering and normalization for library-size differences. We performed statistical comparisons of LAB-S to GA-R and LL to GG. We performed a one-tailed (to account for directionality of gene expression differences) $\chi^{2}$ test to examine whether the overlap of differentially expressed (DE) genes was more than expected by chance.

In addition to analyzing global differential expression, we used the RNA-seq data to better understand gene structure and expression within the candidate QTL. For each sample, we used Trinity (Haas et al. 2013) to construct de novo transcriptome assemblies for each sample. Using tblastn, we identified the transcripts in each Trinity assembly corresponding to all StringTie transcripts from the region from bp 4,370,000-4,620,000. We then took the longest transcript 
352 from each Trinity assembly and used orfipy (Singh and Wurtele 2021) to extract the longest open

353 reading frame (ORF) for each gene. Next we compared the ORFs from each of the 12 samples,

354 looking for differences in predicted protein structure between the samples with resistant

355 chromosome 13 genotypes (GA-R and GG) and susceptible ones (LAB-S and LL). We

356 quantified midgut abundance of transcripts for all genes expressed in this region with average log

357 count per million reads across all samples produced by edgeR. We used PROVEAN (Choi and

358 Chan 2015) to predict the effects of amino acid substitutions between LAB-S and GA-R for each

359 of the genes in this region.

360 After identifying a nonsense mutation in the kinesin-12 gene, we manually inspected this site in

361 IGV (Robinson et al. 2017). After performing Sanger sequencing of the GA strain and field

362 samples (see below), we additionally inspected this site by visualizing sequencing

363 chromatograms in Teal (Rausch et al. 2020). We analyzed the putative kinesin-12 protein

364 computationally using blastp to find homologous proteins. We then used blastp to compare the

365 sequence conservation upstream and downstream of the stop codon mutation in the lepidopteran

366 species H. armigera (XP_021193241.1), Chloridea virescens (PCG76683.1), Spodoptera litura

367 (XP_022828947.1), Manduca sexta (KAG6448083.1), and Bombyx mori (XP_004927959). We

368 used a two-tailed paired t-test to assess amino acid conservation across these species before and

369 after the nonsense mutation. We aligned these sequences with both the GA-R and LAB-S H. zea

370 sequences with Clustal Omega (Sievers and Higgins 2018) and plotted the alignments using

371 TeXshade (Beitz 2000). To further probe the potential structure and function of the protein, we

372 used InterProScan 5 (Jones et al. 2014) to search for additional protein domains, AlphaFold 2.1.0

373 (Jumper et al. 2021) to predict the 3D protein structure, SignalP 5.0 (Armenteros et al. 2019) to

374 examine if the protein contained signal peptides, and DeepGOPlus (Kulmanov and Hoehndorf

375 2020) to predict gene ontology (GO; Ashburner et al. 2000) categorization based on the

376 sequence.

\section{Genotyping of the GA strain and field samples for the kinesin-12 mutation}

379 We collected H. zea larvae from the field in Maricopa, Arizona in October 2020 and Tifton, 
380 Georgia in July 2021. Both populations had high resistance to Cry1Ac (Yu et al. 2021; Y.

381 Carrière, unpubl. data). We reared larvae to adults in the lab and collected tissue from 25 of the

382 Maricopa samples and 39 of the Tifton samples. We extracted DNA using a squish extraction

383 (Gloor et al. 1993) in $150 \mu$ l of buffer. We analyzed five F72 GA individuals sequenced in

384 October 2016 (Fritz et al. 2020). We downloaded raw reads from NCBI (PRJNA599999),

385 trimmed them using Trimmomatic, aligned them to the GA-R genome with bwa-mem, and

386 identified the frequency of the C546T mutation with samtools and VarScan. We additionally

387 collected tissue from 20 F87 adults from the GA strain in May 2018 and extracted DNA with

388 Qiagen DNeasy Blood and Tissue Kits. We designed primers (Supplementary Table S2) to

389 amplify the region surrounding the kinesin-12 mutation causing a stop codon. We performed

390 PCR as above, although with the addition of final concentration $0.1 \mu \mathrm{g} / \mu \mathrm{l}$ bovine serum albumin

391 (Sigma-Aldrich, St. Louis, MO, USA) due to PCR inhibitors. Sanger sequencing was done at

392 Eurofins Genomics as described above. We screened sequences manually in Geneious Prime

393 (Biomatters, Auckland, NZ) for presence of the target mutation.

Analysis of 11 genes previously implicated in lepidopteran resistance to Cry1Ac

We used our results from GWAS and RNA-seq to test the hypothesis that one or more of 11 genes previously implicated in lepidopteran resistance to Cry1Ac contributed to resistance in our strains. For each gene, we present the lowest $P$-value from the original GWAS of SNPs between

399 the start and end of the gene in the $\mathrm{G}^{\prime}$ analysis. We also report the FDR corrected $P$-values from 400 differential expression analyses in edgeR comparing GA-R versus LAB-S and within GA-RS 401 comparing individuals with both alleles from GA-R (GG) versus those with both alleles from 402 LAB-S (LL). 
We generated a de novo chromosome-level assembly of the genome of the GA-R strain of H. zea with 31 chromosomes, $375.2 \mathrm{Mb}, 36.9 \%$ GC content, $33.0 \%$ repeat content, an N50 scaffold length of $12.9 \mathrm{Mb}$, and 15,482 encoded proteins (Supplementary Table S4). Of 5,286 conserved single-copy lepidopteran genes, this genome has $98.9 \%$ complete, $98.5 \%$ complete and singlecopy, $0.4 \%$ duplicated, $0.3 \%$ fragmented, and only $0.8 \%$ missing. The new genome assembly has only one gap set to $100 \mathrm{bp}$, which occurs in chromosome 17 and represents $0.000027 \%$ of the genome. This is a considerable improvement from the H. zea assembly of Pearce et al. (2017), which has $34.1 \mathrm{Mb}$ of gaps representing $10 \%$ of that genome (Supplementary Table S4). Relative

414 to the previous $H$. zea genome assembly, the new assembly has 64-fold greater N50, 10\% more 415 base pairs, $15 \%$ more complete BUSCO genes, and double the repeat content (Supplementary 416 Table S4). Relative to previous estimates based on bacterial artificial chromosome sequencing 417 and flow cytometry, the new genome size is 3\% larger than an estimate for $\mathrm{H}$. zea (Coates et al. 418 2017) and 5\% smaller than an estimate for H. armigera (Zhang et al. 2019b).

419 The 31 chromosomes in the new assembly are largely syntenic with those of $H$. armigera (Pearce 420 et al. 2017; Valencia-Montoya et al. 2020), although with different inversion karyotypes for 19 421 chromosomes (Supplementary Figure S1). We also found substantial differences between H. zea

422 and $H$. armigera in the $\mathrm{Z}$ chromosome (chromosome 1) that are not associated with reversed 423 sequences and thus probably not caused by chromosomal inversions (Supplementary Figure S1).

424 An alternative hypothesis is that errors in one or both assemblies contributed to these differences.

425 Errors are less likely in the new H. zea assembly because of its higher N50 and lower gap 426 percentage relative to the H. armigera assembly (Supplementary Table S4, Pearce et al. 2017).

427 In particular, the $\mathrm{Z}$ chromosome assembled cleanly without additional merging in the new $H$. zea 428 assembly.

Genomic comparison between GA-R and the susceptible strain LAB-S

431 Sequencing of 30 larvae from GA-R and 30 larvae from the unrelated susceptible strain LAB-S 432 revealed 165,416 fixed differences between the strains, as well 941,146 variable sites in GA-R 433 and 911,946 in LAB-S. Analysis of Tajima's D showed many regions with low genetic variation 434 throughout the genome in both strains (Supplementary Figures S2 and S3). For both strains, 
435

these regions could reflect genetic drift or selective sweeps caused by adaptation to lab conditions. For GA-R, regions of low variation could also reflect selection for resistance in the lab.

\section{Genome-Wide Association Study (GWAS) of resistance to Cry1Ac in GA-RS}

We created the GA-RS strain by crossing GA-R and LAB-S. Both the Z-score and G' sliding window analyses of 1,578,733 SNPs from the GWAS using pools of resistant and susceptible larvae from the F12 generation of GA-RS identified a region associated with resistance on chromosome 13 (Figure 1, Supplementary Figure S4). G’ analysis via QTLseqr defined this QTL as a region from 4.0 to $6.5 \mathrm{Mb}$. This QTL contains 117 SNPs associated with resistance at $P<5$ $\times 10^{-8}$. All of these 117 SNPs showed the expected relationship with the parental strain. The 108 alleles found at higher frequency in the resistant larvae were more common in GA-R than LAB$\mathrm{S}$. The remaining nine alleles were at higher frequency in the susceptible larvae and were more common in LAB-S than GA-R.

Outside of this QTL, only 18 SNPs were associated with resistance at $P<5 \times 10^{-8}$. Both chromosome 10 and 15 had two of these SNPs. No other chromosome had more than one. In the $\mathrm{G}^{\prime}$ analysis, only the QTL on chromosome 13 was significantly associated with resistance (Supplementary Figure S4). We refer to this QTL as the $r 1$ locus.

Analysis of Z-scores from the GWAS shows that SNP sites that differed significantly between resistant and susceptible larvae were not evenly distributed across the chromosome 13 QTL (Figure 2A). Eighteen of the 25 windows of $10 \mathrm{~kb}$ with the top 5\% significant SNP density were clustered between 4.42 and $4.60 \mathrm{Mb}$. Consistent with the GWAS results, Tajima's D provides evidence for a selective sweep in GA-R between 4.3 and $5.2 \mathrm{Mb}$ on chromosome 13 (Figure 2B). 
461 GA-RS for SNPs at 12 marker sites within $r 1$. This revealed eight markers (2 to 9) from 4.3 to $4625.0 \mathrm{Mb}$ significantly associated with resistance (Table 1). For marker 4 at $4.5 \mathrm{Mb}(\mathrm{n}=57)$ and 463 marker 5 at $4.6 \mathrm{Mb}(\mathrm{n}=60)$, all resistant larvae genotyped from GA-RS were either homozygous 464 for the allele from the resistant GA-R strain (GG) or heterozygous, with one allele from GA-R 465 and the other from the susceptible LAB-S strain (GL) (Table 1). These results were confirmed via Sanger sequencing for all 60 resistant individuals and 34 susceptible individuals. A similar test using only resistant larvae from the F26 confirmed this result: all 23 resistant larvae genotyped were either GG (16) or GL (7). By contrast, the three genotypes were in HardyWeinberg equilibrium in 89 larvae genotyped from a control sample from the F26 that was not screened for resistance and thus contained a mixture of resistant and susceptible individuals (24 GG: 44 GL: $\left.21 \mathrm{LL}, \chi^{2}=0.0091 ; P=0.52\right)$. Genotype frequency in the F26 larvae differed significantly between the resistant larvae and the control larvae $\left(\chi^{2}=22.60, P=1.2 \times 10^{-5}\right)$.

The results from the GWAS, Tajima's D, the G' analysis, analysis of SNP density, and fine-scale mapping (Figures 1 and 2, Table 2), identify the region between 4.3 to $4.6 \mathrm{Mb}$ as most likely to contain the mutation(s) causing the effect of chromosome 13 on resistance to Cry1Ac. This

476 region is captured by a single contig in both our Canu and DBG2OLC assemblies

477 (Supplementary Figure S5) and is syntenic with a region of the H. armigera chromosome 13 478 (Supplementary Figures S1 and S6).

481 Based on the results above and annotations from funannotate and StringTie, we focused on the 48210 genes between 4.37 and 4.62 Mb on chromosome 13. Six of these 10 genes were expressed 483 substantially in the midgut of third instar larvae (Figure 3A, Table 2). The most highly expressed 484 gene encodes a wild-type protein of 308 amino acids that has sequence identity of $97 \%$ with 485 kinesin-related protein 12 in H. armigera (XP_021193241; Supplementary Figure S7, 486 Supplementary Table S5). The structure of this gene in terms of introns and exons is also similar 487 between $H$. zea and H. armigera (Supplementary Figure S6B). Hereafter, we refer to this gene in 
In GA-R we found a point mutation (C546T) in kinesin-12 that introduces a premature stop codon expected to truncate the protein at 183 amino acids (Figure 3B, Supplementary Figure S7).

491 Manual inspection of aligned genomic reads, RNA-seq reads, and Sanger sequences further

492 confirmed the identity of the SNP (Supplementary Figures S7, S8 and S9). This mutation

493 occurred in $100 \%$ of reads covering the SNP from 30 GA-R larvae and in $0 \%$ of reads covering

494 the SNP from 30 LAB-S larvae that were sequenced in the genomic comparison between these

495 strains. In the GWAS with GA-RS, this mutation was more common in resistant larvae (71\%)

496 than susceptible larvae $\left(32 \% ; P=7.48 \times 10^{-6}\right)$. It occurs at bp 4,547,246, between the two

497 markers (4 and 5) most tightly associated with resistance to Cry1Ac (Table 1). Furthermore,

498 RNA-seq near marker 4 detected the C546T mutation in 100\% of reads covering the SNP from

$49915 \mathrm{GG}$ larvae and 0\% of reads covering the SNP from 15 LL larvae, confirming complete

500 linkage between this mutation and marker 4. All of this evidence identifies the C546T mutation

501 in kinesin-12 as a candidate for causing the contribution of the $r l$ allele to resistance to Cry1Ac.

502 Aside from the kinesin-12 mutation, we detected missense mutations between GA-R and LAB-S

503 linked to marker 4 in three of the other six candidate genes in this region that were expressed

504 substantially in midguts of third instar larvae. These genes encode juvenile hormone esterase,

505 phosphatidylinositol 4-phosphate 3-kinase, and ubiquitin protein ligase (Table 2). However,

506 according to PROVEAN, none of the amino acid substitutions in these genes are expected to

507 have major effects on protein function.

508 Although the kinesin-12 gene has been annotated as encoding a kinesin-related protein in $H$.

509 armigera, both its wild-type function and the effects of the nonsense mutation remain unclear.

510 Within the moth family Noctuidae, amino acid sequence identities relative to the LAB-S strain of

$511 H$. zea are $97 \%$ for $H$. armigera (as noted above), $87 \%$ for $C$. virescens, and $61 \%$ for S. litura

512 (Supplementary Table S5). Outside this family, no annotated proteins in Lepidoptera have

513 greater than $45 \%$ amino acid identity and we found no orthologs in other insect orders. For five

514 species of Lepidoptera, including the three mentioned above plus B. mori and M. sexta, the

515 sequence identity for this protein relative to LAB-S did not differ significantly between upstream

516 and downstream from the stop codon ( $\mathrm{t}_{4}=0.95, P=0.40$; Supplementary Table S5). Thus, we

517 cannot reject the null hypothesis that evolutionary constraints are similar for the portions of the

518 protein before and after the stop codon. 
519 Analysis with SignalP found no evidence for a signal peptide, indicating this protein is not likely

520 to be integrated into or secreted through the cell membrane. The most specific of the GO terms

521 associated with this protein by DeepGoPlus (Supplementary Table S6) is intracellular non-

522 membrane-bounded organelle, which is most closely associated with kinesin-related proteins in

523 Drosophila melanogaster (http://amigo.geneontology.org/amigo/term/GO:0043232).

524 InterProScan and AlphaFold identified a coil with high confidence (aa 124-194; Supplementary

525 Figures S11 and S12) that would be disrupted in the truncated form of the protein. Thus, we find

526 moderate evidence the $H$. zea kinesin-12 gene encodes a kinesin protein whose function might be 527 disrupted by the C546T nonsense mutation.

\section{Kinesin-12 mutation in the GA strain and in field samples}

530 To test the hypothesis that the C546T mutation in kinesin-12 originated in the field, we

531 determined its frequency in the GA strain of $H$. zea, which was selected for resistance in the

532 field, but not in the lab (Brévault et al. 2013; Welch et al. 2015). In GA, the frequency of the

533 C546T mutation was 0.80 in five larvae from the F72 generation (three with homozygous TT

534 genotypes and two with heterozygous CT genotypes), which does not differ significantly from its

535 frequency of 0.975 in 20 larvae from the F87 generation (19 homozygous TT and one

536 heterozygous CT; Fisher's exact test: $P=0.10)$. These results are consistent with the hypothesis

537 that C546T mutation originated in the field population from which GA was established. The

538 alternative hypothesis that this mutation was absent in the field and arose in the lab seems

539 unlikely. Based on the mean of ca. 900 moths per generation for GA and assuming a mutation

540 rate of $3 \times 10^{-9}$ per nucleotide site (Keightley et al. 2015; Yoder and Tiley 2021), the probability

541 of a single mutation arising at a particular nucleotide site in GA during 72 generations is 0.0004 .

542 The high frequency of C546T in GA after rearing for many generations in the lab without

543 exposure to Bt toxins implies this mutation caused little or no fitness cost when reared in the lab

544 in the absence of Bt toxins. However, we did not find this mutation in 39 individuals collected

545 from the field in Georgia in July 2021 or in 25 individuals derived from the field in Arizona in

546 2020, despite the resistance to Cry1Ac in both of these field populations (Yu et al. 2021; Y. 
547 Carrière, unpubl. data). Whereas all individuals from the Georgia 2021 sample had the same

548 sequence as LAB-S at the codon starting with bp 546, three individuals from Arizona had a

549 single base pair substitution (C546A) changing the encoded amino acid from glutamine to lysine.

550 In the field samples from Arizona in 2020 and Georgia in 2021, we detected no insertions,

551 deletions, or other mutations introducing a stop codon in the $200 \mathrm{bp}$ upstream or downstream

552 from the C546T mutation.

\section{Inheritance and trajectory of resistance in GA-RS}

555 The genotype frequencies at marker 4 in resistant and susceptible larvae indicate at least one

556 GA-R allele at this locus was necessary for resistance in our screening bioassay at $10 \mu \mathrm{g}$ Cry1 Ac

557 per $\mathrm{cm}^{2}$ diet (Table 1). However, $27 \%$ of susceptible larvae were homozygous for the GA-R

558 allele at marker 4 (Table 1). Together these results suggest that the $r l$ allele was necessary but

559 not sufficient for resistance to Cry1Ac in our screening bioassay.

560 Compared to 89 control larvae reared on untreated diet, 69 resistant larvae from the F22, F23, 561 and F26 generations had a significantly higher ratio of the GG genotype to the GL genotype for

562 marker 4 (Fisher's exact test; $P<0.0001$ ). Based on the data for marker 4 from the F22, F23, and

563 F26, the $r 1$ allele had a value for dominance $(h)$ of 0.23 (Supplementary Table S3), which is

564 intermediate between completely recessive inheritance $(h=0)$ and additive inheritance $(h=0.5)$.

565 To test the hypothesis that alleles at one or more other loci contributed to resistance, we

566 compared the trajectory of resistance based on bioassay data with the trajectory of the GA-R

567 allele at marker 4. Resistance to Cry1Ac decreased substantially over time (Figure 4;

568 Supplementary Table S1), indicating that in the absence of Cry1Ac, a pleiotropic fitness cost was 569 associated with one or more alleles contributing to resistance. However, the frequency of the

570 GA-R allele at marker 4 was 0.52 in 89 control larvae from the F26, which is not different than

571 the expected 0.50 in the F1. This suggests no fitness cost was associated with the $r 1$ allele, which

572 is tightly linked with GA-R allele at marker 4. In the F26, marker 4 was in Hardy-Weinberg

573 equilibrium as noted above, confirming the absence of selection at this locus in GA-RS. The

574 significant decrease in resistance over time despite no decline in the frequency of the GA-R 
575

576

577

578

579

580

allele at marker 4 implies the decrease in resistance was caused by reduced frequency of one or more resistance alleles that carry a fitness cost in the absence of Cry1 Ac and are not linked with the $r 1$ allele.

\section{Analysis of gene expression using RNA-seq}

To test the hypothesis that differential gene expression contributes to resistance, we used RNAseq to compare transcript levels between GA-R and LAB-S and between the GG and LL genotypes within GA-RS. After filtering, we analyzed expression of 12,965 genes (Supplementary Table S7). We found 2,173 differentially expressed (DE) genes between the unrelated strains LAB-S and GA-R (Supplementary Table S8) and 23 DE genes between the GG and LL genotypes within GA-RS (Supplementary Table S9). None of the genes associated with $r l$ in chromosome 13 differed significantly in expression between GA-R and LAB-S or between GG and LL.

Twelve of the $23 \mathrm{DE}$ genes between GG and LL were also among the set of 2,173 DE genes in the parental strain comparison, of which nine were DE in the same direction in both comparisons (higher expression in GA-R than LAB-S and in GG than LL or vice versa; Tables S6 and S7). The overlap in DE genes between these two datasets is significantly greater than expected by chance $\left(\chi^{2}=3.86 ; P=0.025\right)$, implying the within-strain comparison between GG and LL reflects meaningful differences between the parental strains. However, none of the $23 \mathrm{DE}$ genes between GG and LL (Supplementary Table S9) are among the 11 genes previously implicated in resistance to Cry $1 \mathrm{Ac}$ in lepidopterans (Table 3). One gene significantly downregulated in both GA-R and GG is on chromosome 1 and encodes a sodium/potassium/calcium exchanger (Supplementary Tables S8 and S9). This transmembrane protein has some functional similarities to $\mathrm{ABC}$ transporters and could be a candidate as a $\mathrm{Bt}$ receptor. However, expression was reduced only 2.6-fold in GG versus LL and 2.5-fold in GA-R versus LAB-S. Together these results indicate the $r l$ region exerts a trans-regulatory effect on gene expression, but the current data provide no compelling evidence that any difference in expression influences resistance. 


\section{Analysis of 11 genes previously implicated in lepidopteran resistance to Cry1Ac}

604 We used our results from GWAS and RNA-seq to evaluate potential contributions to resistance

605 of 11 genes previously implicated in lepidopteran resistance to Cry1Ac (Table 3). None of these

606 candidate genes are in the resistance-associated QTL on chromosome 13 or the putative minor

607 effect QTL on chromosome 10 (Table 3). In addition, none of them had any SNPs that were

608 significantly associated with resistance in the GWAS (Table 3). Although tetraspanin-1 is on

609 chromosome 10 in H. zea, it is outside the regions of this chromosome that were moderately

610 associated with resistance in the GWAS. As noted above, none of the 23 DE genes between GG

611 and LL are among the 11 candidate genes (Supplementary Table S9). Only one of the 11

612 pairwise comparisons between strains based on RNA-seq showed a significant difference.

613 Expression of $A B C C 1$ was significantly lower in GA-R than LAB-S $(P=0.0014$, Table 3; Table

614 S6). However, within GA-RS, expression of $A B C C 1$ did not differ significantly between GG and

615 LL $(P=0.29$; Table 3$)$, which indicates reduced expression of $A B C C 1$ was not genetically linked

616 with resistance conferred by $r l$.

619 We report three key results demonstrating a novel genetic basis of Cry1Ac resistance in the GA-

$620 \mathrm{R}$ strain of $H$. zea, which resulted from field selection followed by lab selection (Brévault et al.

621 2013; Welch et al. 2015). First, resistance was associated with a 250-kb region of chromosome

62213 that contains no genes with a previously identified role in Bt resistance or toxicity. Second,

623 within this region, resistance to Cry1Ac was associated with a point mutation that introduces a

624 premature stop codon in a novel candidate gene, kinesin-12. Third, we report evidence that one

625 or more other loci also contributed to resistance to Cry1Ac. To facilitate these advances, we built

626 the first chromosome-level genome assembly for H. zea, adding to a growing set of highly

627 contiguous genomes for lepidopteran pests (Chen et al. 2019b; Ward et al. 2021; Yan et al.

628 2021). This genome was essential for the genetic mapping reported here and will serve as a

629 resource for other genomic investigations into the biology of $H$. zea.

630 Our findings add a new candidate gene to the diverse list of genes associated with Bt resistance 
631 in lepidopterans (Jin et al. 2018; Guo et al. 2021; Jurat-Fuentes et al. 2021). However, the novel

632 genetic basis of resistance does not necessarily imply a novel biochemical mechanism of

633 resistance. The effects of $r l$ could be mediated by either decreased toxin activation or reduced

634 binding of Cry1Ac to larval midgut membranes, which are well known mechanisms of Bt

635 resistance (Peterson et al. 2017; Jurat-Fuentes et al. 2021). Indeed, previous studies of $H$. zea

636 have found decreased protoxin activation in GA-R (Zhang et al. 2019) and reduced binding of

637 Cry1Ac to larval midgut preparations in strains with resistance caused by knocking out the

638 putative receptor ABCC2 (Perera et al. 2021).

639 The location of $r l$ on chromosome 13 is noteworthy because it corresponds closely to the region

640 under the strongest selection in H. zea populations from Louisiana that were exposed to Bt crops

641 over the past 19 years (Taylor et al. 2021). Although Taylor et al. (2021) identified a narrow

642 region near but not containing $r l$ as the most likely site of selection ( 4.0 Mb in our assembly),

643 the broader region associated with the selective sweep in their data includes $r 1$ ( 3.8 to $5.8 \mathrm{Mb})$

644 and aligns with both our original and refined QTL for resistance. Thus, $r 1$ might be associated

645 with resistance to Cry $1 \mathrm{Ab}$ (which is similar to Cry1 Ac) in the field populations of H. zea from

646 Louisiana studied by Taylor et al. (2021), as well as in GA-R and its parent strain GA (Fritz et

647 al. 2020), which originated from a field-selected population in Georgia in 2008 (Brévault et al.

648 2013).

649 The RNA-seq evidence does not support the hypothesis that altered transcription in the $r 1$ region

650 causes resistance. Thus, a protein-coding mutation is more likely to be responsible for the

651 contribution of the $r 1$ region to resistance. We hypothesize that this contribution is mediated by

652 the premature stop codon in the kinesin-12 gene. Among the protein-coding mutations in the

653 candidate region, only this nonsense mutation that shortens the predicted protein by $40 \%$ is

654 expected to have a major effect on protein function. Furthermore, of the 10 genes in the region

655 tightly associated with resistance, midgut expression was highest for kinesin-12, suggesting a

656 midgut function for the protein it encodes. Protein functional prediction algorithms including

657 DeepGoPlus provide moderate support for the original annotation as a kinesin with a function in

658 intracellular transport or structure. Nonetheless, we do not know the normal function of the

659 kinesin-12 protein and cannot infer that the C546T mutation causes resistance. In future work,

660 we aim to test the hypothesis that the C546T mutation contributes to resistance by determining if 
661 resistance is reduced when we use CRISPR/Cas9 to replace the mutant sequence in GA-R with

662 the wild type sequence from LAB-S (Jin et al. 2018; Fabrick et al. 2021). If disruption of the

663 kinesin-12 gene is not sufficient for resistance, as the results here imply, we expect that knocking

664 out this gene would not cause resistance in a susceptible strain.

665 Kinesins and kinesin-related proteins are motor proteins important in microtubule function,

666 chromosomal movement, and organelle transport (Ali and Wang 2020) that have not been

667 associated previously with Bt resistance. Several kinesins are involved in mitogen-activated

668 protein kinase (MAPK) signaling cascades (Liang and Yang 2019) and MAPK signaling is

669 implicated in Bt resistance (Guo et al. 2015, 2020, 2021; Qin et al. 2021). Furthermore, a case of

670 xenobiotic resistance in mice involved a mutant kinesin acting downstream of a MAPK (Watters

671 et al. 2001). Thus, one hypothesis is that kinesin-12 acts downstream of MAPK as part of the

672 signaling cascade initiated when Cry1 Ac binds to a midgut receptor. However, MAPK

673 influences Bt resistance via downregulation of Bt receptors (Guo et al. 2015, 2020, 2021; Qin et

674 al. 2021). Here we see no evidence for reduced transcription of putative receptors, making this

675 an unlikely explanation for the link between kinesin-12 and resistance.

676 Kinesins also play a role in the localization of transmembrane proteins to the cell membrane

677 (Jana et al. 2021) and in intracellular cadherin trafficking (Phang et al. 2014). The transport

678 functions of kinesins and kinesin-related proteins entail motor complexes of three or more

679 proteins (Phang et al. 2014), suggesting interactions between different proteins could be

680 interrupted to interfere with receptor transport to the membrane. We hypothesize that in the GA-

$681 \mathrm{R}$ strain of $H$. zea, a truncated kinesin-12 in combination with mutations affecting one or more of

682 its interacting partners blocks proper localization of a $\mathrm{Bt}$ receptor on the membrane.

683 The results from GWAS and fine-scale mapping show the $r l$ allele was necessary, but not

684 sufficient for resistance in our screening bioassay, implying contributions from one or more

685 additional loci. If a second unlinked mutation were also necessary for resistance in our screening

686 bioassay, this would be expected to yield a second major peak in the GWAS, similar to the peak

687 for the QTL in chromosome 13. The lack of a second major peak suggests that mutations in two

688 or more unlinked loci could each cause resistance in combination with $r l$ (e.g., $r l$ plus mutation

$689 X$ or $r l$ plus mutation $Y$ could cause resistance). 
690 The decline in resistance over time in GA-RS also implies more than one locus contributed to

691 resistance. While resistance to Cry1Ac declined significantly across generations in GA-RS, the

692 frequency of C546T and other $r 1$ linked alleles did not. After 22, 23 and 26 generations without

693 exposure to Bt toxins, it was not lower than its expected initial frequency of 0.50 . Thus, the

694 decline in resistance reflects a decreased resistance allele frequency at one or more other loci.

695 Unlike the C546T mutation, which did not have a substantial fitness cost in the lab, the observed

696 decline in resistance suggests that a fitness cost was associated with at least one mutation at

697 another locus that contributed to resistance in GA-RS. Polygenic resistance to Cry1Ac or

698 Cry1 Ab also has been reported in strains of $H$. zea unrelated to GA-R (Caccia et al. 2012;

699 Lawrie et al. 2020; Perera et al. 2021; Taylor et al. 2021) and in other species of Lepidoptera

700 (Kaur and Dilwari 2010; Zhao et al. 2021; Ma et al. 2022).

701 The results summarized above have implications for understanding the trajectory of the C546T

702 mutation in the field. The high frequency of the C546T mutation in the field-selected GA strain

703 that was started with 180 field-collected larvae suggests this mutation was common in 2008 in

704 the moderately resistant field population in Georgia from which GA was derived (Brévault et al.

705 2013). In the absence of exposure to Cry1Ac, the frequency of this mutation did not increase

706 significantly in GA from the F72 to F87 or in GA-RS from the expected frequency in the F1 to

707 the observed frequency in the F26. Thus, because GA was not exposed to Cry1Ac in the lab, it is

708 unlikely the frequency of this mutation was low initially in GA and subsequently increased

709 because of strong selection. Nonetheless, we did not detect this mutation in Cry1Ac-resistant

710 populations from the same site in Georgia in 2021 or in Arizona in 2020. Thus, this mutation is

711 not associated with resistance to Cry1Ac in all field populations of $H$. zea. Also, its frequency

712 apparently decreased in the field in Georgia from 2008 to 2021. One hypothesis is that the

713 frequency of this mutation decreased in Georgia because it has a substantial fitness cost under

714 field conditions, such as reduced overwintering survival (Carrière et al. 2001), which would not

715 be evident in the lab. The C546T mutation could have been replaced by one or more mutations

716 that have lower fitness costs (Guillemaud et al. 1998), confer higher resistance to Cry1Ac, and/or

717 confer resistance without contributions from mutations at other loci. More research is needed to

718 determine the function of kinesin-12 and its role in resistance, as well as the genetic basis of

719 Cry1Ac resistance in current field populations of H. zea. 
722 All raw sequence data is available at NCBI (Bioproject: PRJNA767434). Phenotypic data, HRM

723 and Sanger genotyping data, initial and final genome assemblies, genome annotations, and

724 scripts for analyses and figures are all available via OSF. Supplementary materials are available

725 at figshare.

\section{Acknowledgments}

728 Mention of trade names or commercial products in this article is solely for the purpose of

729 providing specific information and does not imply recommendation or endorsement by the U.S.

730 Department of Agriculture. USDA is an equal opportunity provider and employer. We thank

731 Xinzhi Ni for sending H. zea from Georgia; Alex Yelich and Chandran Unnithan for help with

732 insect rearing and dissections; Yidong Wu, David Heckel, Megan Fritz, Katherine Taylor, Fred

733 Gould, and Juan Luis Jurat-Fuentes for their valuable comments on the manuscript; and Jon

734 Galina-Mehlman, Jayson Talag, and Dave Kudrna for their assistance and advice regarding

735 PacBio and Illumina sequencing.

738 J.F., Y.C., B.E.T., and L.M.M. conceived and designed the project. K.M.B., C.W.A., B.A.D., 739 and X.L. performed experiments and collected the data. K.M.B., C.W.A., Y.C., and B.E.T.

740 analyzed data. K.M.B. and B.E.T. wrote the manuscript with input from all authors.

\section{$742 \quad$ Funding}

743 This work was supported by grants from the USDA National Institute of Food and Agriculture 744 (Agriculture and Food Research Initiative 2020-67013-31924 and Biotechnology Risk 
Assessment Research Grants Program 2020-33522-32268), Corteva Agriscience, and the Cotton

746 Insect Resistance Management (IRM) Technical Subcommittee of the Agricultural

747 Biotechnology Stewardship Technical Committee (ABSTC).

\section{Conflicts of interest}

750 As noted above, support for this study was provided in part by Corteva Agriscience and the 751 Cotton IRM Technical Subcommittee of the ABSTC.

\section{Literature cited}

Ali MI, Luttrell RG, Young III SY. 2006. Susceptibilities of Helicoverpa zea and Heliothis virescens (Lepidoptera: Noctuidae) populations to Cry1Ac insecticidal protein. J Econ Entomol. 99:164-175.

Ali I, Yang W-C. 2020. The functions of kinesin and kinesin-related proteins in eukaryotes. Cell Adh Migr. 14: 139-152.

Anilkumar KJ, Rodrigo-Simón A, Ferré J, Pusztai-Carey M, Sivasupramaniam S, et al. 2008.

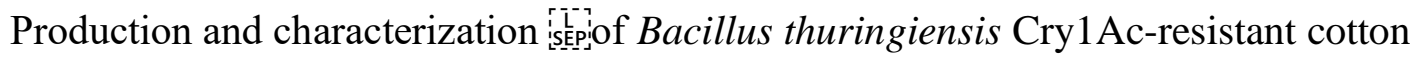
bollworm Helicoverpa zea (Boddie). Appl Environ Microbiol. 74:462-469.

Armenteros JJA, Tsirigos KD, Sønderby CK, Peterson TN, Winther O, et al. 2019. SignalP 5.0 improves signal peptide predictions using deep neural networks. Nat Biotech. 37:420423.

Ashburner M, Ball CA, Blake JA, Botstein D, Butler H, et al. 2000. GeneOntology: tool for the unification of biology. Nat Genet. 25:25-29.

Barsh GS, Copenhaver GP, Gibson G, Williams SM. 2012. Guidelines for genome-wide association studies. PLoS Genet. 8:e1002812. 
Bacillus thuringiensis toxin resistance in Lepidoptera. Genetics. 189:675-679.

Beitz E. 2000. TeXshade: shading and labeling of multiple sequence alignments using LaTeX2e. Bioinformatics. 16:135-139.

Benowitz KM, Coleman JM, Matzkin LM. 2019. Assessing the architecture of Drosophila mojavensis locomotor evolution with bulk segregant analysis. G3 (Bethesda). 9:17671775.

Bolger AM, Lohse M, Usadel B. 2014. Trimmomatic: a flexible trimmer for Illumina sequence data. Bioinformatics. 30:2114-2120.

Bravo A, Likitvivatanavong S, Gill SS, Soberón M. 2011. Bacillus thuringiensis: a story of a successful bioinsecticide. Ins Biochem Mol Biol. 41:423-431.

Brévault T, Heuberger S, Zhang M, Ellers-Kirk C, Ni X, et al. 2013. Potential shortfall of pyramided transgenic cotton for insect resistance management. Proc Natl Acad Sci USA. 110:5806-5811.

Brévault T, Tabashnik BE, Carrière Y. 2015. A seed mixture increases dominance of resistance to Bt cotton in Helicoverpa zea. Sci Rep. 5:9807.

Caccia S, Moar WJ, Chandrashekhar J, Oppert C, Anilkumar KJ, et al. 2012. Association of Cry1Ac toxin resistance in Helicoverpa zea (Boddie) with increased alkaline phosphatase levels in the midgut lumen. Appl Env Microbiol. 78:5690-5698.

Calles-Torrez V, Knodel JJ, Boetel MA, French BW, Fuller BW, et al. 2019. Field-evolved resistance of northern and western corn rootworm (Coleoptera: Chrysomelidae) populations to corn hybrids expressing single and pyramided Cry3Bb1 and Cry34/35Ab1 Bt proteins in North Dakota. J Econ Entomol. 112:1875-1886.

\section{Carrière Y, Ellers-Kirk C, Patin AL, Sims M, Meyer S, et al. 2001. Overwintering cost} associated with resistance to transgenic cotton in the pink bollworm. J. Econ. Entomol. 94:935-941.

Carrière Y, Crickmore N, Tabashnik BE. 2015. Optimizing pyramided transgenic Bt crops for sustainable pest management. Nat Biotechnol. 33:161-168. seasonal changes in cotton plants on the evolution of resistance to pyramided cotton producing the Bt toxins Cry1Ac and Cry1F in Helicoverpa zea. Pest Man Sci. 74:627637. 
802

803

804

805

806

807

808

Carrière Y, Degain B, Unnithan GC, Harpold VS, Li X, et al. 2019. Seasonal declines in Cry1 Ac and $\mathrm{Cry} 2 \mathrm{Ab}$ concentration in maturing cotton favor faster evolution of resistance to pyramided Bt cotton in Helicoverpa zea. J Econ Entomol. 112:2907-2914.

Carrière, Y, Brown Z, Aglasan S, Dutilleul P, Carroll M, et al. 2020a. Crop rotation mitigates impacts of corn rootworm resistance to transgenic Bt corn. Proc Natl Acad Sci USA. $117: 18385-18392$.

Carrière Y, Degain BA, Harpold VS, Unnithan GC, Tabashnik BE. 2020b. Gene flow between $\mathrm{Bt}$ and non-Bt plants in a seed mixture increases dominance of resistance to pyramided $\mathrm{Bt}$ corn in Helicoverpa zea (Lepidoptera: Noctuidae). J Econ Entomol. 113:2041-2051.

Carrière Y, Degain BA, Tabashnik BE. 2021. Effects of gene flow between Bt and non-Bt plants in a seed mixture of Cry1A.105 + Cry2Ab corn on performance of corn earworm in Arizona. Pest Manag Sci. 77: 2106-2113

Chakraborty M, Baldwin-Brown JG, Long AD, Emerson JJ. 2016. Contiguous and accurate de novo assembly of metazoan genomes with modest long read coverage. Nuc Ac Res. 44: e147.

Chen L, Wei J, Liu C, Niu L, Zhang C, et al. 2019a. Effect of midgut specific binding protein ABCC1 on Cry1Ac toxicity against Helicoverpa armigera. Sci Ag Sin. 52:3337-3345.

Chen W, Yang X, Tetreau G, Song X, Coutu C, et al. 2019b. A high-quality chromosome-level genome assembly of a generalist herbivore, Trichoplusia ni. Mole Ecol Res. 19:485-496.

Chin CS, Alexander DH, Marks P, Klammer AA, Drake J, et al. 2013. Nonhybrid, finished microbial genome assemblies from long-read SMRT sequencing data. Nat Methods. 10:563-569.

Choi Y, Chan AP. 2015. PROVEAN web server: a tool to predict the functional effect of amino acid substitutions and indels. Bioinformatics. 31: 2745-2747.

Coates BS, Abel CA, Perera OP. 2017. Estimation of long terminal repeat element content in the Helicoverpa zea genome from high-throughput sequencing of bacterial artificial chromosome pools. Genome. 60:310-324.

Cook DR, Threet M. 2019. Cotton insect losses - 2019. https://www.entomology.msstate.edu/resources/2019loss.php

Dively GP, Venugopal PD, Bean D, Whalen J, Holmstrom K, et al. 2018. Regional pest suppression associated with widespread Bt maize adoption benefits vegetable growers. 
Proc Natl Acad Sci USA. 115:3320-3325.

Fabrick JA, LeRoy DM, Mathew LG, Wu Y, Unnithan GC, et al. 2021. CRISPR-mediated mutations in the $\mathrm{ABC}$ transporter gene $\mathrm{ABCA} 2$ confer pink bollworm resistance to $\mathrm{Bt}$ toxin Cry2Ab. Sci Rep. 11:10377.

Ferguson KB, Kursch-Metz T, Verhulst EC, Pannebakker BA. 2020. Hybrid genome assembly and evidence-based annotation of the gg parasitoid and biological control agent Trichogramma brassicae. G3 (Bethesda). 10:3533-3540.

Flynn JM, Hubley R, Goubert C, Rosen J, Clark AG, et al. 2020. RepeatModeler2 for automated

Fritz ML, Nunziata SO, Guo R, Tabashnik BE, Carrière Y. 2020. Mutations in a novel cadherin gene associated with Bt resistance in Helicoverpa zea. G3 (Bethesda). 10:1563-1574.

Gahan LJ, Gould F, Heckel DG. 2001. Identification of a gene associated with Bt resistance in Heliothis virescens. Science 293:857-860.

Gloor GB, Preston CR, Johnson-Schlitz DM, Nassif NA, Phillis RW, et al. 1993. Type I repressors of $P$ element mobility. Genetics. 135:81-95.

Guillemaud T, Lenormand T, Bourguet D, Chevillon C, Pasteur N, et al. 1998. Evolution of resistance in Culex pipiens: allele replacement and changing environment. Evolution. 52:443-453.

Guo Z, Kang S, Chen D, Wu Q, Wang S, et al. 2015. MAPK signaling pathway alters expression of midgut ALP and ABCC genes and causes resistance to Bacillus thuringiensis Cry1Ac toxin in diamondback moth. PLoS Genetics. 11:e1005124.

Guo Z, Kang S, Sun D, Gong L, Zhou J, et al. 2020. MAPK-dependent hormonal signaling plasticity contributes to overcoming Bacillus thuringiensis toxin action in an insect host. Nat Commun. 11:3003.

Guo Z, Kang S, Wu Q, Wang S, Crickmore N, et al. 2021. The regulation landscape of MAPK signaling cascade for thwarting Bacillus thuringiensis infection in an insect host. PLoS Path. 17:e1009917.

Haas BJ, Papanicolau A, Yassour M, Grabherr M, Blood PD, et al. 2013. De novo transcript sequence reconstruction from RNA-seq using the Trinity platform for reference generation and analysis. Nat Prot. 8:1494-1512. 
Hartke J, Schell T, Jongepier E, Schmidt H, Sprenger PP, et al. 2019. Hybrid genome assembly of a neotropical mutualistic ant. Genom Biol Evol. 11:2306-2311.

Heckel DG, Gahan LJ, Baxter SW, Zhao J-Z, Shelton AM, et al. 2007. The diversity of Bt resistance genes in species of Lepidoptera. J Inv Biol. 95:192-197.

ISAAA. 2019. Global status of commercialized biotech/GM crops: 2019. ISAAA Brief No. 55. ISAA: Ithaca, NY.

Jaworski CC, Allan CW, Matzkin LM. 2020. Chromosome-level hybrid de novo genome assemblies as an attainable option for nonmodel insects. Mol Ecol Res. 20:1277-1293.

Jin L, Wei Y, Zhang L, Yang Y, Tabashnik BE, et al. 2013. Dominant resistance to Bt cotton and minor cross-resistance to Bt toxin in Cry2 Ab in cotton bollworm from China. Evol Appl. 6:1222-1235.

Jones P, Binns D, Chang HY, Fraser M, Li W, et al. 2014. InterProScan 5: genome-scale protein function classification. Bioinformatics. 30:1236-1240.

Jumper J, Evans R, Pritzel A, Green T, Figurnov M, et al. 2021. Highly accurate protein structure prediction with AlphaFold. Nature. 596:583-589.

Jurat-Fuentes JL, Karumbaiah L, Jakka SRK, Ning C, Liu C, et al. 2011. Reduced levels of membrane-bound alkaline phosphatase are common to lepidopteran strains resistant to Cry toxins from Bacillus thuringiensis. PLoS One. 6:e17606.

Jurat-Fuentes JL, Heckel DG, Ferré J. 2021. Mechanisms of resistance to insecticidal proteins from Bacillus thuringiensis. Annu Rev Entomol. 66:121-140.

Kajitani R, Toshimoto K, Noguchi H, Toyoda A, Ogura Y, et al. 2014. Efficient de novo assembly of highly heterozygous genomes from whole-genome shotgun short reads. Genom Res. 24:1384-1395.

Karsch-Mizrachi I, Nakamura Y, Cochrane G. 2012. The International Nucleotide Sequence Database Collaboration. Nuc Ac Res. 40:D33-D37.

Kaur P, Dilwari VK. 2011. Inheritance of resistance to Bacillus thuringiensis Cry1Ac toxin in Helicoverpa armigera (Hübner) (Lepidoptera: Noctuidae) from India. Pest Man Sci. 67:1294-1302. 
895

896

897

898

899

900

901

902

903

904

905

906

907

908

909

910

Kaur G, Guo J, Brown S, Head GP, Price PA, et al. 2019. Field-evolved resistance of Helicoverpa zea (Boddie) to transgenic maze expressing pyramided Cry1A.105.Cry2Ab2 proteins in northeast Louisiana, the United States. J Inv Pathol. 163:11-20.

Keightley PD, Pinharanda A, Ness RW, Simpson F, Dasmahapatra KK et al. 2015. Estimation of the spontaneous mutation rate in Heliconius melpomene. Mol Biol Evol. 32:239-243.

Kim D, Paggi JM, Park C, Bennett C, Salzberg SL. 2019. Graph-based genome alignment and genotyping with HISAT2 and HISAT-genotype. Nat Biotech. 37:907-915.

Kofler R, Orozco-terWengel P, De Maio N, Pandey RV, Nolte V, et al. 2011. PoPoolation: a toolbox for population genetic analysis of next generation sequencing data from pooled individuals. PLoS One. 6:e15925.

Koren S, Walenz BP, Berlin K, Miller JR, Bergman NH, et al. 2017. Canu: scalable and accurate long-read assembly via adaptive k-mer weighting and repeat separation. Genom Res. 27:722-736.

Kulmanov M, Hoehndorf R. 2020. DeepGOPlus: improved protein function prediction from sequence. Bioinformatics. 36:422-429.

Langmead B, Salzberg S. 2012. Fast gapped-read alignment with Bowtie 2. Nat Methods. 9:357359.

Lawrie RD, Mitchell III RD, Deguenon JM, Ponnusamy L, Reisig D, et al. 2020. Multiple known mechanisms and a possible role of an enhanced immune system in Bt-resistance in a field population of the bollworm, Helicoverpa zea: differences in gene expression with RNAseq. Int J Mol Sci. 21:6528.

Lawrie RD, Mitchell III RD, Deguenon JM, Ponnusamy L, Reisig D, et al. 2022. Characterization of long non-coding RNAs in the bollworm, Helicoverpa zea, and their possible role in Cry1Ac-resistance. Insects. 13:12.

Li H. 2011. A statistical framework for SNP calling, mutation discovery, association mapping and population genetical parameter estimation from sequencing data. Bioinformatics. 27:2987-2993.

Li H, Durbin R. 2009. Fast and accurate short read alignment with Burrows-Wheeler transform. Bioinformatics. 25:1754-1760.

Liang Y-J, Yang W-X. 2019. Kinesins in MAPK cascade: how kinesin motors are involved in the MAPK pathway? Gene. 684:1-9. 
Liu Y, Tabashnik BE. 1997. Inheritance of resistance to the Bacillus thuringiensis toxin Cry1C in the diamondback moth. Appl Env Microbiol. 63:2218-2223.

Liu C, Xiao Y, Li X, Oppert B, Tabashnik BE, et al. 2014. Cis-mediated down-regulation of a trypsin gene associated with Bt resistance in cotton bollworm. Sci Rep. 4:7219. larvae attacking cotton and soybean to purified endotoxin proteins and commercial formulations of Bacillus thuringiensis. J Econ Entomol. 92:21-32.

Ma W, Zhao X, Yin C, Jian F, Du X, et al. 2020. A chromosome-level genome assembly reveals the genetic basis of cold tolerance in a notorious rice insect pest, Chilo suppressalis. Mol Ecol Res. 20:268-282.

Ma X, Shao E, Chen W, Cotto-Rivera RO, Yang X, et al. 2022. Bt Cry1Ac resistance in Trichoplusia $n i$ is conferred by multi-gene mutations. Insect Biochem Mol Biol. 140:103678.

Magwene PM, Willis JH, Kelly JK. 2011. The statistics of bulk segregant analysis using next generation sequencing. PLoS Comp Biol. 7:e1002255.

Mansfeld BN, Grumet R. 2018. QTLseqr: an R package for bulk segregant analysis with nextgeneration sequencing. Plant Genom. 11:180006.

Mathers TC. 2020. Improved genome assembly and annotation of the soybean aphid (Aphis glycines Matsumura). G3 (Bethesda). 10: 899-906.

Musser FR, Catchot AL, Conley SP, Davis JA, DiFonzo C, et al. 2019. 2018 Soybean insect losses in the United States. Midsouth Entomol. 12: 1-24.

National Academies of Sciences, Engineering, and Medicine. 2016. Genetically Engineered Crops: Experiences and Prospects. Washington DC, National Academies Press. nutrient intake by Helicoverpa zea (Lepidoptera: Noctuidae) larvae. J Econ Entomol. 108:2581-2588. 
Palmer JN, Stajich J. 2020. Funannotate v1.8.1: Eukaryotic genome annotation (v1.8.1). Zenodo. https://doi.org/10.5281/zenodo.4054262.

Patro R, Duggal G, Love MI, Irizarry RA, Kingsford C. 2017. Salmon provides fast and biasaware quantification of transcript expression. Nat Meth. 14:417-419.

Pearce SL, Clarke DF, East PD, Elfekih S, Gordon KHJ, et al. 2017. Genomic innovations, transcriptional plasticity and gene loss underlying the evolution and divergence of two highly polyphagous and invasive Helicoverpa pest species. BMC Biol. 15:63.

Perera OP, Little NS, Abdelgaffar H, Jurat-Fuentes JL, Reddy GVP. 2021. Genetic knockouts indicate that the ABCC2 protein in the bollworm Helicoverpa zea is not a major receptor for the Cry1Ac insecticidal protein. Genes. 12:1522.

Pertea M, Pertea GM, Antonescu CM, Chang TC. Mendell JT, et al. 2015. StringTie enables improved reconstruction of a transcriptome from RNA-seq reads. Nat Biotech. 33:290295.

Peterson B, Bezuidenhout CC, Van den Berg J. 2017. An overview of mechanisms of Cry toxin resistance in lepidopteran insects. J Econ Entomol. 110:362-377.

Qin J, Guo L, Ye F, Kang S, Sun D, et al. 2021. MAPK-activated transcription factor PxJun

Rajagopal R, Arora N, Sivakumar S, Rao NGV, Nimbalkar SA, et al. 2009. Resistance of Helicoverpa armigera to Cry1 Ac toxin from Bacillus thuringiensis is due to improper processing of the protoxin. Biochem J. 419:309-316.

Rausch T, Fritz MH-Y, Untergasser A, Benes V. 2020. Tracy: basecalling, alignment, assembly and deconvolution of sanger chromatogram trace files. BMC Genom. 21:230.

Reisig DD, Huseth AS, Bacheler JS, Aghaee M-A, Braswell L, et al. 2018. Long-term and observational evidence of practical Helicoverpa zea resistance to cotton with pyramided Bt toxins. J Econ Entomol. 111:1824-1833.

Reisig DD, DiFonzo C, Dively G, Fargan Y, Gore F, et al. 2021. Best management practices to delay the evolution of $\mathrm{Bt}$ resistance in Lepidopteran pests without high susceptibility to Bt toxins in North America. J Econ Entomol. https://doi.org/10.1093/jee/toab247. 
applications. Nat Genet. 46:912-918.

Roach MJ, Schmidt SA, Borneman AR. 2018. Purge Haplotigs: allelic contig reassignment for third-gen diploid genome assemblies. BMC Bioinf. 19:460.

Robinson MD, McCarthy DJ, Smythe GK. 2010. edgeR: a Bioconductor package for differential expression analysis of digital gene expression. Bioinformatics. 26:139-140.

Robinson JT, Thorvaldsdóttir H, Wenger AM, Zehir A, Mesirov JP. 2017. Variant review with the Integrative Genomics Viewer. Cancer Res. 77:31-34.

Romeis J, Naranjo SE, Meissle M, Shelton AM. 2018. Genetically engineered crops help support conservation biological control. Biol Contr. 130:136-154.

Schmidt H, Hellmann SL, Waldvogel A-M, Feldmeyer B, Hankeln T, et al. 2020. A high-quality genome assembly from short and long reads for the non-biting midge Chironomus riparius (Diptera). G3 (Bethesda). 10:1151-1157.

Seppey M, Manni M, Zdobnov EM. 2019. BUSCO: assessing genome assembly and annotation completeness. In: Kollmar M (ed.) Gene Prediction. Methods in Molecular Biology, vol 1962. Humana, New York, NY. pp. 227-245.

Sievers F Higgins DG. 2018. Clustal Omega for making accurate alignments of many protein sequences. Prot Sci. 27:135-145.

Singh U, Wurtele ES. 2021. orfipy: a fasta and flexible tool for extracting ORFs. Bioinformatics. 37:3019-3020.

Smit AFA, Hubley R, Green P. 2013-2015. RepeatMasker Open-4.0. http://repeatmasker.org

1010 Smith JL, Farhan Y, Schaafsma AW. 2019. Practical resistance of Ostrinia nubilalis (Lepidoptera: Crambidae) to Cry1F Bacillus thuringiensis maize discovered in Nova

1013 Soberón M, Pardo-López L, López I, Gómez I, Tabashnik BE, et al. 2007. Engineering modified Bt toxins to counter insect resistance. Science. 318:1640-1642.

1015 Soneson C, Love MI, Robinson MD. 2016. Differential analyses for RNA-seq: transcript-level 1016 estimates improve gene-level inferences. F100Research. 4:1521.

1017 Tabashnik BE and Carrière Y. 2019. Global patterns of resistance to Bt crops highlighting pink 
1019 Tabashnik BE, Liu Y-B, Malvar T, Heckel DG, Masson L, et al. 1998. Insect resistance to Bacillus thuringiensis: uniform or diverse? Phil Trans R Soc B. 353:1751-1756.

Tabashnik BE, Gassmann AJ, Crowder DW, Carrière Y. 2008. Insect resistance to Bt crops: evidence versus theory. Nat Biotech. 26:199-202.

Tabashnik BE, Liesner LR, Ellsworth PC, Unnithan GC, Fabrick JA, et al. 2021. Genetically engineered cotton synergizes eradication of the pink bollworm a century after its invasion of the United States. Proc Natl Acad Sci USA. 118:e2019115118.

Taylor KL, Hamby KA, DeYonke AM, Gould F, Fritz ML. 2021. Genome evolution in an agricultural pest following adoption of transgenic crops. Proc Natl Acad Sci USA. 118:e2020853118.

U.S. Dept. of Agriculture, Agricultural Marketing Service. Cotton Varieties Planted 2008 Crop. https://apps.ams.usda.gov/Cotton/AnnualCNMarketNewsReports/VarietiesPlanted/2008-

U.S. Dept. of Agriculture, Economic Research Service. 2020. Adoption of Genetically Engineered Crops in the U.S. https://www.ers.usda.gov/data-products/adoption-ofgenetically-engineered-crops-in-the-us/ (accessed 3 December 2021).

Valencia-Montoya WA, Elfekih S, North HL, Meier JI, Warren IA, et al. 2020. Adaptive introgression across semipermeable species boundaries between local Helicoverpa zea and invasive Helicoverpa armigera moths. Mol Biol Evol. 37:2568-2583. VarietiesPlanted.pdf https://www.ams.usda.gov/mnreports/cnavar.pdf (accessed 3 December 2021).

Walker BJ, Abeel T, Shea T, Priest M, Abouelliel A, et al. 2014. Pilon: an integrated tool for comprehensive microbial variant detection and genome assembly improvement. PLoS One. 9:e112963.

1047 Ward CM, Perry KD, Baker G, Powis K, Heckel DG, et al. 2021. A haploid diamondback moth (Plutella xylostella L.) genome assembly resolves 31 chromosomes and identifies a diamide resistance mutation. Ins Biochem Mol Biol. 138:103622. 
1050 Watters JW, Dewar K, Lehoczky J, Boyartchuk V, Dietrich WF. 2001. Kif1C, a kinesin-like motor protein, mediates mouse macrophage resistance to anthrax lethal factor. Curr Biol. 11: $1503-1511$.

Welch KL, Unnithan GC, Degain BA, Wei J, Zhang J, et al. 2015. Cross-resistance to toxins used in pyramided Bt crops and resistance to Bt sprays in Helicoverpa zea. J Invert Pathol. 132:149-156.

Welter D, MacArthur J, Morales J, Burdett T, Hall P, et al. 2014. The NHGRI GWAS Catalog, a curated resource of SNP-trait associations. Nuc Ac Res. 42:D1001-D1006.

Xu H, Zhao X, Yang Y, Chen X, Mei Y, et al. 2021. Chromosome-level genome assembly of an agricultural pest, the rice leaffolder Cnaphalocrocis exigua (Crambidae, Lepidoptera). Mol Ecol Res. 21:561-572.

Yan B, Ou H, Wei L, Wang X, Yu X, et al. 2021. A chromosome-level genome assembly of Ephestia elutella (Hübner, 1796) (Lepidoptera: Pyralidae). Genom Biol Evol. 13:evab114.

Ye C, Ma ZS. 2016. Sparc: a sparsity-based consensus algorithm for long erroneous sequencing reads. PeerJ. 4:e2016.

Ye C, Hill CM, Wu S, Ruan J, Ma ZS. 2016. DBG2OLC: efficient assembly of large genomes using long erroneous reads of the third generation sequencing technologies. Sci Rep. $6: 31900$.

Yoder AD, Tiley GP. 2021. The challenge and promise of estimating the de novo mutation rate from whole-genome comparisons among closely related individuals. Mol Ecol. 30:60876100.

Yu W, Lin S, Dimase M, Niu Y, Brown S, et al. 2021. Extended investigation of field-evolved resistance of the corn earworm Helicoverpa zea (Lepidoptera: Noctuidae) to Bacillus thuringiensis Cry1A.105 and Cry2Ab2 proteins in the southeastern United States. J Inv Pathol. 183: 107560

Zhang S, Cheng H, Gao Y, Wang G, Liang G, et al. 2009. Mutation of an aminopeptidase N gene is associated with Helicoverpa armigera resistance to Bacillus thuringiensis Cry1 Ac toxin. Ins Biochem Mol Biol. 39:421-429.

Zhang M, Wei J, Ni X, Zhang J, Jurat-Fuentes JL, et al. 2019a. Decreased Cry1Ac activation by midgut proteases associated with Cry1Ac resistance in Helicoverpa zea. Pest Man Sci. 
75:1099-1106.

1082 Zhang S, Gu S, Ni X, Li X. 2019b. Genome size reversely correlates with host plant range in 1083 Helicoverpa species. Front Physiol. 10:29.

1084 Zhang S, Shen S, Peng J, Zhou X, Kong X, et al. 2020. Chromosome-level genome assembly of 1085 an important pine defoliator, Dendrolimus punctatus (Lepidoptera; Lasiocampidae). Mol

1086 Ecol Res. 20:1023-1037.

1087 Zhao S, Jiang D, Wang F, Tabashnik BE, Wu Y. 2021. Independent and synergistic effects of 1088 knocking out two ABC transporter genes on resistance to Bacillus thuringiensis toxins 1089 Cry1Ac and Cry1Fa in diamondback moth. Toxins. 13:9. 
1092 Table 1 Genotype and allele frequencies at 12 markers on the chromosome 13 QTL for resistant 1093 and susceptible GA-RS larvae from generations F22 and F23.

1094

\begin{tabular}{|c|c|c|c|c|c|c|}
\hline \multirow[b]{2}{*}{ Marker } & \multirow[b]{2}{*}{ Bp (Chr13) } & \multicolumn{2}{|c|}{ Resistant larvae } & \multicolumn{2}{|c|}{ Susceptible larvae } & \multirow[b]{2}{*}{$P$-value ${ }^{b}$} \\
\hline & & $\begin{array}{c}\text { Genotypes } \\
(\mathrm{GG} / \mathrm{GL} / \mathrm{LL})^{\mathrm{a}}\end{array}$ & $\begin{array}{l}\text { G allele } \\
\text { frequency }\end{array}$ & $\begin{array}{c}\text { Genotypes } \\
(\mathrm{GG} / \mathrm{GL} / \mathrm{LL})^{\mathrm{a}}\end{array}$ & $\begin{array}{l}\text { G allele } \\
\text { frequency }\end{array}$ & \\
\hline 1 & $4,110,560$ & $41 / 15 / 4$ & 0.81 & $23 / 23 / 3$ & 0.70 & 1.0 \\
\hline 2 & $4,281,434$ & $30 / 23 / 7$ & 0.69 & $9 / 24 / 16$ & 0.43 & $9.6 \mathrm{e}-03$ \\
\hline 3 & $4,379,538$ & $41 / 18 / 1$ & 0.83 & $13 / 24 / 12$ & 0.51 & $2.4 \mathrm{e}-04$ \\
\hline 4 & $4,475,706$ & $40 / 17 / 0$ & 0.85 & $13 / 24 / 12$ & 0.51 & $4.0 \mathrm{e}-05$ \\
\hline 5 & $4,596,970$ & $43 / 17 / 0$ & 0.86 & $13 / 24 / 12$ & 0.51 & $3.0 \mathrm{e}-05$ \\
\hline 6 & $4,720,567$ & $39 / 17 / 2$ & 0.82 & $12 / 13 / 12$ & 0.50 & $1.3 \mathrm{e}-04$ \\
\hline 7 & $4,829,519$ & $40 / 18 / 1$ & 0.83 & $13 / 23 / 13$ & 0.50 & $1.0 \mathrm{e}-04$ \\
\hline 8 & $4,902,478$ & $38 / 21 / 1$ & 0.81 & $13 / 23 / 13$ & 0.50 & $1.0 \mathrm{e}-04$ \\
\hline 9 & $4,998,799$ & $38 / 19 / 1$ & 0.82 & $12 / 23 / 14$ & 0.48 & $5.0 \mathrm{e}-05$ \\
\hline 10 & $5,226,319$ & $27 / 19 / 3$ & 0.75 & $14 / 24 / 5$ & 0.61 & 0.47 \\
\hline 11 & $5,727,295$ & $11 / 22 / 7$ & 0.55 & $5 / 14 / 1$ & 0.60 & 0.29 \\
\hline 12 & $6,232,397$ & $15 / 19 / 8$ & 0.58 & $3 / 33 / 8$ & 0.44 & 1.0 \\
\hline
\end{tabular}

${ }^{a} \mathrm{G}$ indicates the allele was more common in the resistant GA-R strain, $\mathrm{L}$ indicates the allele was more common in the susceptible LAB-S strain.

${ }^{\mathrm{b}}$ From Fisher's exact test of the null hypothesis that allele frequency did not differ between the resistant and susceptible larvae. 
1096 Table 2 Larval midgut expression of genes in the region of chromosome 13 QTL associated with 1097 resistance to Cry1Ac.

1098

\begin{tabular}{|c|c|c|c|}
\hline Gene ID & Start - Stop (orientation) & Name & $\begin{array}{c}\text { Larval midgut } \\
\text { expression } \\
\text { (mean } \log _{2} \mathrm{CPM} \text { ) }\end{array}$ \\
\hline MSTRG.8092 & $4,371,598-4,491,148(+)$ & $\begin{array}{c}\text { Cyclic AMP-response element- } \\
\text { binding protein A }\end{array}$ & 2.44 \\
\hline MSTRG.8095 & $4,491,241-4,520,601(-)$ & $\begin{array}{c}\text { Heparan-alpha-glucosaminide- } N \text { - } \\
\text { acetyltransferase }\end{array}$ & $-0.02 *$ \\
\hline MSTRG.8097 & $4,504,492-4,507,681(+)$ & $\begin{array}{l}\text { Juvenile hormone esterase-like } \\
\text { Carboxyl/cholinesterase CCEO06D }\end{array}$ & 4.84 \\
\hline hz_G0000107 & $4,536,278-4,541,251(+)$ & Lipase member $H$-B-like & N/A \\
\hline MSTRG.8100 & $4,541,250-4,593,328(-)$ & $\begin{array}{c}\text { Phosphatidylinositol 4-phosphate 3- } \\
\text { kinase C2 domain-containing } \\
\text { subunit alpha }\end{array}$ & 3.25 \\
\hline MSTRG.8101 & $4,546,116-4,547,856(+)$ & Kinesin-related protein 12-like & 7.08 \\
\hline hz_G0000111 & $4,593,482-4,595,264(-)$ & Uncharacterized protein & N/A \\
\hline hz_G0000112 & $4,600,520-4,606,939(+)$ & Uncharacterized protein & N/A \\
\hline MSTRG.8102 & $4,607,861-4,617,975(-)$ & Ubiquitin-protein ligase E3A & 3.88 \\
\hline MSTRG.8103 & $4,618,419-4,620,800(+)$ & $\begin{array}{c}\text { Retinal rod rhodopsin-sensitive } \\
\text { cGMP 3`, 5-cyclic } \\
\text { phosphodiesterase subunit delta }\end{array}$ & 2.98 \\
\hline
\end{tabular}

1100 Gene IDs refer to the StringTie annotation when expressed for correspondence with the RNA1101 seq data. Funannotate IDs refer to non-expressed genes. N/A indicates the gene was not 1102 expressed. *Because of the low expression of this gene (12-fold lower than the median for the 1103 other six expressed genes), we considered it not to be substantially expressed. 
1105 Table 3 GWAS and RNA-seq results for 11 genes previously implicated in lepidopteran 1106 resistance to Cry1Ac.

1107

\begin{tabular}{|c|c|c|c|c|c|c|}
\hline Gene & Key reference & $\begin{array}{l}\text { H. zea genome } \\
\text { location }^{\mathrm{a}}\end{array}$ & $\begin{array}{l}\text { Gene } \\
\text { ID }^{\mathrm{b}}\end{array}$ & $\begin{array}{l}\text { GWAS: } \\
P \text {-value }\end{array}$ & $\begin{array}{l}\text { RNA-seq: } \\
\text { GA-R vs. } \\
\text { LAB-S } \\
P \text {-value }\end{array}$ & $\begin{array}{l}\text { RNA-seq: } \\
\text { GG vs. LL } \\
P \text {-value }^{\mathrm{d}}\end{array}$ \\
\hline$A B C C 1$ & Chen et al. 2019a & 12: 9.24-9.29 & 7445 & 0.16 & 0.0014 & 0.29 \\
\hline$A B C C 2$ & Baxter et al. 2011 & 15: 7.07-7.09 & 9800 & 0.35 & 0.95 & 0.56 \\
\hline APN1 & Zhang et al. 2009 & 9: $11.33-11.37$ & 5943 & 0.53 & 0.32 & 0.33 \\
\hline Cadherin & Gahan et al. 2001 & 6: $1.89-1.97$ & 4086 & 0.46 & 1.0 & 0.13 \\
\hline Cadherin-86C & Fritz et al. 2020 & 12: $4.60-4.62$ & 7702 & 0.49 & 0.69 & 0.75 \\
\hline MAPK4 & Guo et al. 2015 & 12: $6.80-6.82$ & 9824 & 0.21 & 0.12 & 0.43 \\
\hline$m A L P$ & Jurat-Fuentes et al. 2011 & 8: $10.41-10.43$ & 4895 & 0.37 & 0.11 & 0.62 \\
\hline Polycalin & Wang et al. 2020 & $25: 2.72-2.73$ & 16287 & 0.48 & 0.73 & 0.14 \\
\hline$S P 2$ & Rajagopal et al. 2009 & $7: 1.34-1.35$ & 4260 & 0.53 & 0.85 & 0.88 \\
\hline Tetraspanin 1 & Jin et al. 2018 & 10: $11.58-11.59$ & 6160 & 0.087 & 0.061 & 0.20 \\
\hline $\operatorname{Try} R$ & Liu et al. 2014 & 27: 2.04-2.09 & 17235 & 0.60 & 0.97 & 1.0 \\
\hline
\end{tabular}

$1109{ }^{\mathrm{b}}$ Full Gene ID begins with MSTRG.

$1110{ }^{\mathrm{c}}$ Lowest $P$-value for any SNP in the gene based on the $\mathrm{G}^{\prime}$ analysis

$111{ }^{d} P$-values are FDR-corrected, bold indicates significant at $<0.05$.

1112

1113

1114 
1116 Figure 1 Manhattan plot of GWAS results showing - $\log _{10}$ of the $P$-values for Z-scores comparing 1117 allele frequencies between resistant and susceptible larvae. The red line indicates the threshold for 1118 significant association $\left(P=5 \mathrm{e}^{-8}\right)$.

Figure 2 Association between resistance to Cry1Ac and SNPs within the chromosome 13 QTL. (A) Proportion of significant SNPs $\left(P<1 \mathrm{e}^{-5}\right)$ from the Z-score analysis of the QTL data in 10-kb sliding windows. The horizontal red line indicates the $95^{\text {th }}$ percentile of the distribution. (B) Evidence of a selective sweep in GA-R from Tajima's D in 50-kb sliding windows. Blue shading covers the QTL from 4.37 to $4.62 \mathrm{Mb}$. The vertical red bars show the locations of markers 4 and 5 (Table 1). The red asterisk indicates the location of kinesin-12.

Figure 3 Ten genes including kinesin-12 in the resistance-associated QTL on chromosome 13. (A) The four genes at the top are in the (-) orientation, the other six below are in the $(+)$ orientation (Table 2). The four genes in white were not expressed substantially in the midgut. Darker red indicates higher expression in the midgut (Table 2). (B) The structure of kinesin-12 in LAB-S and GA-R. Boxes represent exons, light blue indicates UTRs, and dark blue signifies coding regions.

Figure 4 Survival of the heterogeneous GA-RS strain of $H$. zea tested on diet with 1 (red) or 10 (blue) $\mu \mathrm{g}$ Cry1Ac per $\mathrm{cm}^{2}$ diet. Survival at each test concentration decreased significantly. Regressions of percent survival to third instar on generation: $\mathrm{y}=-2.49 \mathrm{x}+88.63, \mathrm{R}^{2}=0.94$, $\mathrm{df}=$ $2, P=0.021$ and $\mathrm{y}=-0.36 \mathrm{x}+11.67, \mathrm{R}^{2}=0.91, \mathrm{df}=3, P=0.0074$ for 1 and $10 \mu \mathrm{g}$ Cry 1 Ac per $\mathrm{cm}^{2}$ diet, respectively. Generation 26 was tested only at the higher concentration. Shaded areas show $95 \%$ confidence intervals. 


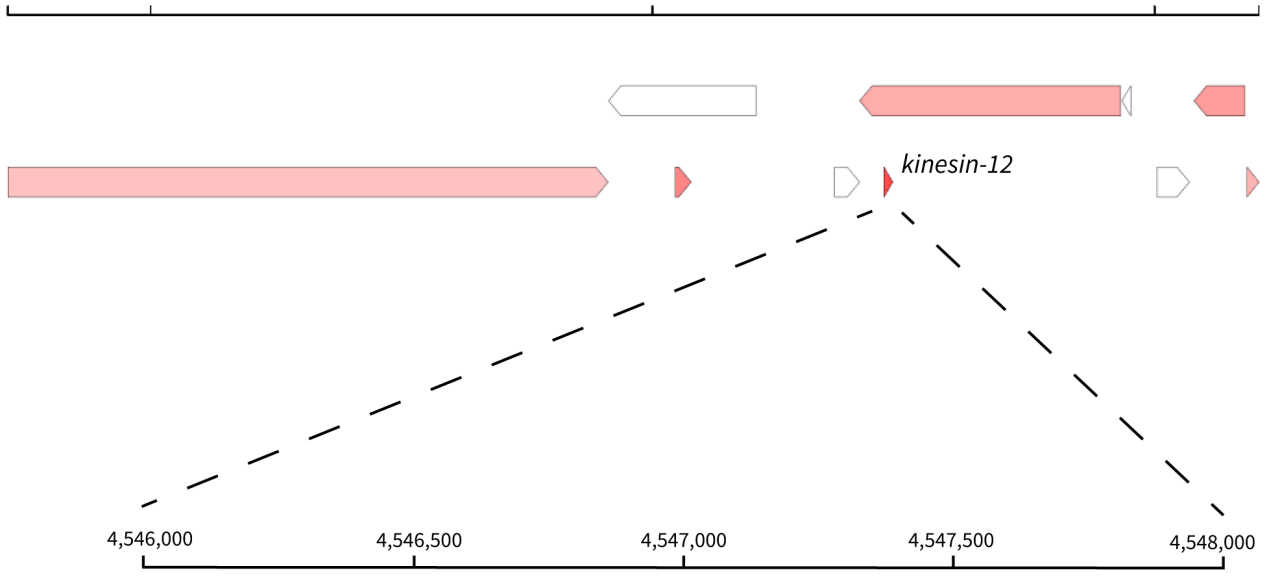

LAB-S

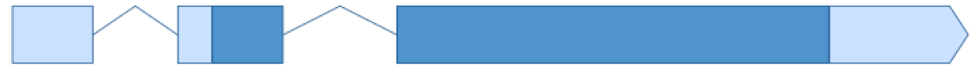

GA-R

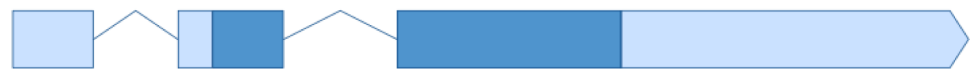




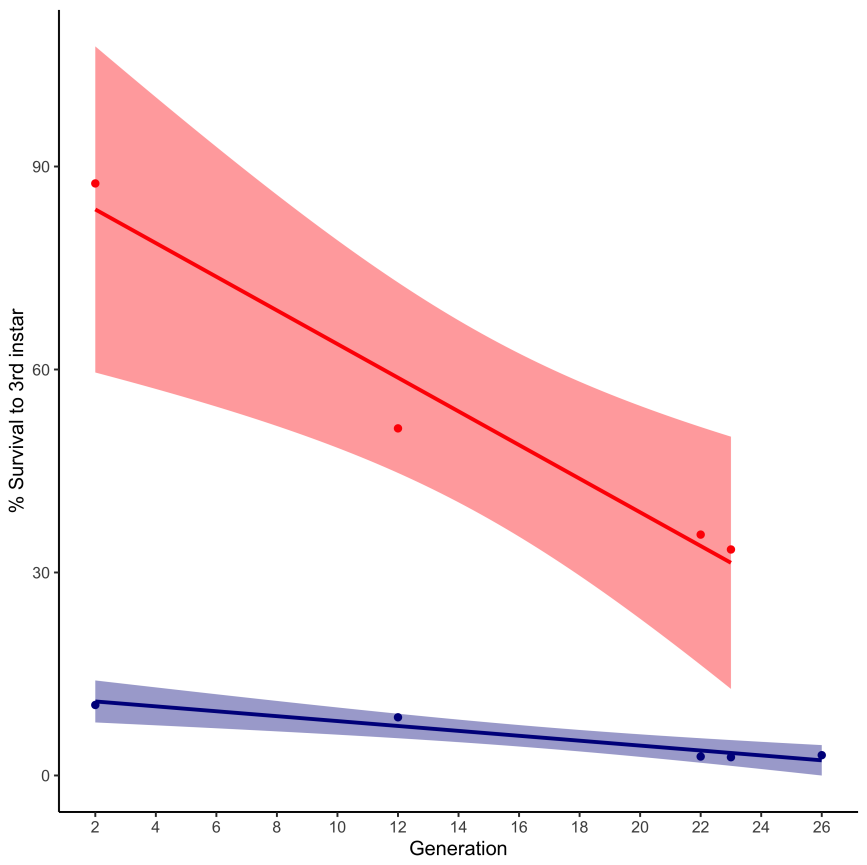

